\title{
Improving Stability of Electronically Controlled Pressure Reducing Valves Through Gain Compensation
}

\author{
Tomasz Janus $^{1}$ and Bogumil Ulanicki ${ }^{2}$ \\ ${ }^{1}$ Independent Researcher, Water Software Systems, De Montfort University, LE1 9BH, Leicester, \\ United Kingdom, Email: tomasz.k.janus@gmail.com \\ ${ }^{2}$ Professor, Water Software Systems, De Montfort University, LE1 9BH, Leicester, United \\ Kingdom, Email: bul@dmu.ac.uk
}

\begin{abstract}
This paper explains the root cause of instabilities which tend to arise in pressure reducing valves (PRVs) under low flow conditions. It was found that the loss of stability in PRVs is a direct result of an increase in the static valve/network gain as the valve position gets smaller, thus making pressure changes more sensitive to valve position adjustments. If the valve controller is tuned at medium valve openings characteristic of normal operating conditions, the increased gain at low valve openings can cause the control system to be too aggressive in its valve position adjustments leading to oscillations. The manuscript provides a mathematical derivation of the gain equation for a simplified pipe-PRV-pipe model. The obtained gain equation curve is then used to derive the formula for a gain compensator whose purpose is to keep the static gain constant across an entire range of permitted valve openings. A simplified network transient model is then used to recreate a real-life PRV instability event and show the remedial effects of the gain compensator.

Keywords: pressure reducing valve, instability, static gain, gain compensator.

\section{INTRODUCTION}

Energy and resource efficient operation of water distribution networks (WDNs) relies on an adequate choice of pressures at critical nodes within the network and an appropriate control of 
these pressures such that they are maintained close to setpoints regardless of the changes in nodal demands. It is up to engineering judgment to select the desired pressure values at controlled nodes in the WDN bearing in mind that a minimum pressure, e.g. 15, is required at the tap to provide an adequately high flow rate and prevent low pressure failures (Ghorbanian et al. 2015) whilst an increase in pressure inside the pipes beyond the required minimum elevates the risk of pipe bursts and leads to higher losses of water from the existing leaks (Thornton and Lambert 2005). An appropriately designed pressure control system within a WDN will provide good setpoint tracking, i.e. pressure values reflect the changes in the setpoint, good disturbance rejection, i.e. nodal pressures are kept close to the setpoint regardless of demand changes, and additionally will not cause large pressure transients in the system (stability). Although all three above requirements are important, stability is crucial for the infrastructure since pressure transients put additional strain on WDN components and, if high enough, can lead to pipe bursts.

Pressure in WDNs is often controlled by pressure reducing valves (PRVs). A PRV is a valve combined with a controller in a form of a feedback control loop, as depicted in Fig. 1. The purpose of this feedback controller is to maintain the outlet pressure at a constant value or to follow a predefined trajectory irrespectively of inlet pressure and flow (demand) variations. The controller receives an error signal $e$ calculated as a difference between the downstream pressure setpoint $H_{d}^{\text {set }}$ and the measured downstream pressure $H_{d}$, and acts on this signal such that the error is minimized, i.e. $H_{d} \approx H_{d}^{\text {set }}$. If $H_{d}<H_{d}^{\text {set }}$ the controller actuates the valve to increase the valve opening and thus the outlet pressure. In the opposite case valve opening is decreased. The valve/WDN system, also referred to as a plant, is subject to unknown disturbances in the form of demand changes, which can be described as variations in orifice areas $\mathbf{A}_{\text {orifice }}$ at the ends of demand nodes. If we remove the feedback the system under study (i.e. the plant plus the controller) is called open-loop, as opposed to full system with feedback which is referred to as closed-loop. This is a typical control system and its properties, in particular stability, should be analyzed in the context of control theory (e.g. Ogata 2010). From the point of view of the technology in which the controller is implemented PRVs can be divided into two categories: hydraulically controlled valves and electrically controlled 
valves. A hydraulic PRV is typically based on a pilot valve loop where the current outlet pressure is compared with the set-point defined by the preload of a spring in the pilot valve (Prescott and Ulanicki 2003). In addition to a hydraulic PRV two types of electronically controlled PRVs are available: (a) a hydraulic PRV such as the one mentioned above in which the set-point is adjusted by an electronic actuator and (b) a standard valve coupled with an electronic feedback controller which directly actuates the valve position. Both valves in the engineering jargon are referred to as electronically controlled PRVs.

Since PRVs are a crucial component of every WDN, pressure control with PRVs has been a widely researched topic. Unfortunately, most of the existing publications on pressure control are focused on steady-state behavior with omission of controller and plant dynamics. A good review of the state of the art in this area is given by Vicente et al. (2015). Traditionally PRVs have been used to maintain a desired pressure immediately downstream of the valve but recently, the so called remote real time control (RRTC) of water distribution networks (WDNs) where the controlled pressure is at a remote node within a network has been of interest to a number of research groups. Campisano et al. (2012) proposed a simple linear controller for real time control (RTC) of a motorized valve using a steady-state model of a WDN. Similar idea was used for planning of remote real time controlled PRVs for the Oppegård municipality in Norway (Berardi et al. 2015). In this case steady-state WDN model incorporated pressure dependent background leakage. The same authors developed a software module called WDNetXL to support their methodology (Giustolisi et al. 2015). The methodology was further refined by Campisano et al. (2016) through inclusion of the criteria for target node selection, allowing multiple valves to control pressure at the same target node and by considering controller calibration methods as well as wireless communication protocols. Page et al. (2016) proposed an innovation to the design of real-time proportional controllers assuming that the information about the flow through the PRV is available. Recently there has been a positive trend to consider and understand dynamic behavior of valve/WDN systems. As an example, Creaco et al. (2017) performed a simulation study of a RTC valve model combined with an unsteady flow simulator with pulsed nodal demands. In this particular simulation study the results showed some 
pressure and flow oscillations but the authors concluded that the steady-state model was a good approximation for the design of the RTC schemes and PRV stability was not discussed in the paper.

Dynamic behavior of PRVs was also tested experimentally, although none of the empirical research based publications known to authors deal with PRVs during unstable operation, only with stable dynamic response to disturbances. Before we move on to further discussions on PRVs' dynamic behavior it is worth pointing out that PRVs alike other dynamic systems may show an oscillatory response (e.g. to incoming pressure waves) but this is not to be confused with unstable response. Stable response may be sinusoidal as long as its amplitude decays in time whilst instability manifests itself with a growing output for a bound input, e.g. a sinusoid of an amplitude growing in time. As pointed out in Janus and Ulanicki (2017) PRV instability is likely to cause pressure transients but itself is a sole result of dynamic properties of the system, as shall be discussed in the later sections of this manuscript. Instabilities are not caused by pressure transients and in fact can be shown (numerically) to occur in rigid-column simulations where pressure transients are not modeled. Dynamic behavior of PRVs was first analyzed by Brunone and Morelli (1999) who measured the response of an automatic control valve to changes in the position of the valve at the confluence node downstream of the PRV. The results show higher pressure oscillations under lower flows than higher flows which supports the findings of this manuscript. The publication also introduces a technique to obtain the flow-rate curve of a valve through unsteady-state tests, which offers better estimation accuracy, especially under lower flows. Meniconi et al. (2015) measured pressure waves produced during partial a partial closure and opening of a valve downstream of a PRV and initially analyzed the waves using a wavelet transform. Experimental studies on dynamic response of PRVs were taken forward in Meniconi et al. (2016) and Meniconi et al. (2017) who performed further laboratory experiments to monitor the behavior of their PRV under different flow conditions. The authors observed pressure oscillations resulting from the movement of the valve element but since the tested range of flows was quite narrow and, as explained and demonstrated through simulation in Ulanicki and Skworcow (2014) and later in Janus and Ulanicki (2017), PRVs tend to become unstable under sufficiently low flows, instability was not observed. 
Since no article has yet discussed specifically the matter of PRV stability whilst the engineering community needs to deal with often disastrous, as shall be briefly shown below, incidents of PRV instability in WDNs, there is an urgent need to understand why such incidents occur and how they can be prevented. Understanding stability is especially important where the valve element is directly actuated by an electronic controller in RTC schemes. There are two potential reasons for the instability in control schemes in general: (a) increase in the value of the system gain (here increase of the static valve/WDN gain due to the nonlinearity of the valve/WDN system against flow) and (b) increase in the value of the delay in the system due to long actuation times or too long a sampling period. Whilst the latter can be avoided by proper system design, this paper focuses on the former, which is caused solely by the property of the valve and the network. The aim of this paper is to explain this phenomenon in a theoretical manner and derive a precise analytical relationship between the valve opening and the static gain of the valve/WDN system.

How severe a PRV instability can be is demonstrated in one such instability event which occurred in a large-scale pressure control scheme installed in a WDN of one of the major cities in the UK. The above-mentioned instability event is illustrated in Fig. 2 which shows how the valve outlet pressure and valve position began to oscillate under low valve openings (and hence low flow conditions) whilst the PRV managed to regain stability later in the day when the flow increased and the valve settled into a larger opening. The above event incurred serious financial and environmental costs as it caused multiple pipe burst across the network resulting in the loss of water and the loss of service to the consumers. Although, in this case the PRV under study was controlled electronically by a programmable logic controller (PLC) the root-cause of instability is common for all types of PRVs, only the remedy will be different for electronically controlled and mechanically controlled PRVs and shall be subjects of future research papers.

The paper is organized as follows. First, the effects of the static valve/WDN gain on feedback control system stability are demonstrated using a classical control root-locus technique applied to a lumped ordinary differential equation (ODE) model designed to qualitatively represent transient behavior of a WDN. Second, a theoretical formula for the static gain of a simplified valve/WDN 
system consisting of an upstream pipe, PRV, and a downstream pipe is derived using implicit function theorem applied to static network equations. Next, 15-min measurements of flows and pressures obtained from a real network are used to calibrate the simplified hydraulic model such that both the model and the real network exhibit the same static hydraulic properties. The calibrated model is then used to generate the static valve/WDN gain vs. valve position curves for different values of the pressure dependency coefficient $\alpha$ in the pressure-dependent demand model. The curve for a chosen value of $\alpha$ is then used to derive the model of the static gain compensator, the purpose of which is to keep the static gain at a constant value for all permitted valve openings. Finally, the simplified network transient model is used to recreate a real-life PRV instability event briefly described above and to show the remedial effects of the gain compensator.

\section{EFFECT OF STATIC GAIN ON STABILITY OF A FEEDBACK SYSTEM}

The aim of this section is to explain the effect of the static gain of the open-loop valve/WDN system on the stability of the closed-loop system bearing in mind that a water distribution network equipped with a PRV is an example of a closed-loop control system (e.g. Ogata 2010) and can be represented in a simplified block-diagram form as in Fig. 1. The physical meaning of the static gain of the valve/WDN system in the context of pressure control is the ratio between the change in the downstream (outlet) pressure $H_{d}$ and the change in the valve position $x$ in steady-state, i.e. $K(x)=d H_{d} / d x$. The static gain depends on the valve opening (operating point) $x$ signifying that the system is nonlinear. In broader terms, the valve/WDN system is nonlinear and distributed, i.e. it is described by nonlinear partial differential equations (PDEs). The approach usually adopted by control engineers in such a situation is to design a controller for a given operating point $x$ using an approximate lumped model described by a linear time invariant (LTI) ordinary differential equation (ODE) or a system of LTI ODEs. Subsequently, the controller parameters are adapted to changes in the operating point, which is referred to as gain scheduling. A generic LTI ordinary differential equation has the the following form:

$$
a_{n} y^{(n)}+a_{n-1} y^{(n-1)}+\ldots+a_{1} y^{(1)}+a_{0} y=b_{m} u^{(m)}+b_{m-1} u^{(m-1)}+\ldots+b_{1} u^{(1)}+b_{0} u
$$


The above equation can be then transformed from differential form in time domain into an algebraic form in the new Laplace $s$ domain using the Laplace transform (e.g. Hazewinkel, M. 1994) and rearranged to produce the ratio between the output and the input. This ratio is called the transfer function and is shown in Eq. 2.

$$
P(s)=\frac{Y(s)}{U(s)}=\frac{b_{m} s^{m}+b_{m-1} s^{m-1}+\ldots+b_{1} s+b_{0}}{a_{n} s^{n}+a_{n-1} s^{n-1}+\ldots+a_{1} s+a_{0}}
$$

The polynomials in the numerator and the denominator can be then factorized using general algebra

$$
P(s)=\frac{b_{m}\left(s-z_{1}\right)\left(s-z_{2}\right) \ldots\left(s-z_{m-1}\right)\left(s-z_{m}\right)}{a_{n}\left(s-p_{1}\right)\left(s-p_{2}\right) \ldots\left(s-p_{n-1}\right)\left(s-p_{n}\right)}
$$

where the roots of the numerator, $z_{1} \ldots z_{m}$, are called zeros of the transfer function and the roots of the denominator, $p_{1} \ldots p_{n}$, are referred to as the poles of the transfer function. The zeros and the poles are either real or appear in complex conjugate pairs. The poles and zeros completely characterize Eq. 1 and hence provide a complete description of the system dynamics. In particular the poles directly define the homogeneous solution of the differential equation, $y_{h}(t)=\sum_{i=1}^{i=n} C_{i} e^{p_{i} t}$, where $C_{i}$ denotes a constant coefficient next to the $i$-th exponential term of the solution. In other words the poles of the transfer function are the eigenvalues of the differential equation. Clearly, if the poles have negative real parts the homogeneous solution decays to zero and the system is stable. If at least one pole has a positive real part the homogeneous solution diverges to infinity and the system is unstable. The poles and the zeros can be plotted in the complex plane where the horizontal axis represents the real parts $\mathfrak{R}(s)$ and the vertical axis represents the imaginary parts $\mathfrak{J}(s)$. The transfer function of the closed-loop system displayed in Fig. 1 is $G_{\text {closed }}(s)=\frac{P(s) C(s)}{1+P(s) C(s)}$, where $P(s)$ is the plant transfer function and $C(s)$ is the controller transfer function. The expression in the numerator is called open loop transfer function $G_{\text {open }}(s)=P(s) C(s)$ and represents the transfer function of the system in the absence of feedback. If the parameters of the plant or the controller change so do the poles of the closed-loop system. If only one parameter is being varied, typically the static gain of the open loop system, the poles of the closed-loop system will move along curves 
in the complex $\mathfrak{R}(s)-\mathfrak{J}(s)$ plane and such a graphical method of stability analysis is called root-locus (Evans 1950). The steady-state gain of the transfer function in Eq. 2 equals $b_{0} / a_{0}$ and corresponds to the static gain of the valve/WDN system $K(x)=d H_{d} / d x$ at a given operation point. As the gain increases these, hopefully originally stable, poles travel along the root-loci and, at some point, depending on the properties of the plant and the controller, may cross the imaginary axis where the closed-loop system becomes unstable. For the purpose of demonstrating how the static open loop gain affects the stability of the closed-loop system using classical control theory the authors developed examples of conceptual lumped models describing the downstream pressure head $H_{d}$ in response to the changes in the valve position $x$. The first model is depicted in Fig. 3 and was formulated based on the physical understanding of one-dimensional unsteady flow. It is hypothesized that the response of downstream pressure to the changes in valve position can be described as a superposition of two responses: (a) inertial response describing acceleration and deceleration of the mass of water under an assumption of zero compressibility, i.e. rigid column model and (b) the transient response, i.e. pressure oscillations due to water compressibility effects. It is also hypothesized that whilst the inertial response is a function of valve position, the transient response is a function of the change of valve position, i.e. the rate of valve closure/opening. Provided that the valve closes with a sufficiently small rate, i.e. $d x / d t \approx 0$ the transient response will be close to zero and the output will exhibit a predominantly inertial character, which is conceptually in agreement with what is observed in practice. The inertial response is modeled with a first order lag element $\frac{K_{1}}{\tau s+1}$ whilst the oscillatory response is described with a canonical second order system $\frac{K_{2} \omega_{n}^{2}}{s^{2}+2 \zeta \omega_{n} s+\omega_{n}^{2}}$, where $\tau$ denotes the system time constant, $\omega_{n}$ denotes the natural frequency and $\zeta$ is the damping ratio (see e.g. Ogata 2010). As an alternative conceptual lumped model of a valve/WDN system we can use a Maxwell model of a mass supported in parallel by a spring and spring with a damper (see Fig. 4). The Maxwell model exhibits similar behavior to the inertial-oscillator model described above - oscillations superimposed on slow inertial response. The transfer function of the Maxwell model between the force applied to mass $m$ and the mass 
position is as follows:

$$
P_{\text {Maxwell }}(s)=\frac{s+\frac{E_{2}}{\eta}}{s^{3}+\frac{E_{2}}{c} s^{2}+\frac{E_{1}+E_{2}}{m} s+\frac{E_{1} E_{2}}{m \eta}}
$$

where $E_{1}$ is the spring constant of the left spring, $E_{2}$ is the spring constant of the right spring, $m$ is the mass of the body supported by the springs and $\eta$ is the viscous friction coefficient of the damper. To visualize the dynamic behavior of the Maxwell model to a unit step change in the external force the following values of model parameters were assumed: $m=4 \mathrm{~kg}, E_{1}=6.60 \mathrm{~N} / \mathrm{m}$, $E_{2}=8.59 \mathrm{~N} / \mathrm{m}$ and $\eta=48.9 \mathrm{~N} \cdot \mathrm{s} / \mathrm{m}$. These values are used as an example and they are not calibrated to any particular valve/WDN system. The step response of the Maxwell model is plotted in Fig. 5 and shows typical effects of inertial and oscillatory components similar to those exhibited by the previously described inertial-oscillator model (see Fig. 6). The root locus plot for the closed-loop feedback control system from Fig. 1 in which the valve/WDN plant is represented by the above Maxwell model with additional actuator inertia is shown in Fig. 7. The control system has four poles marked with crosses and one zero marked with a circle. Three poles come from the Maxwell model and one from the actuator. When the gain increases from zero to infinity the dominant real pole (closest to the imaginary axes) approaches the zero, the fast actuator real pole goes to 'real' negative infinity whilst the two remaining complex poles diverge to the 'complex' infinity. The closed-system loses stability at the moment where the static gain $K$ reaches the value of 1.41 at which point the two conjugate complex poles cross from left half plane (LHP) to right half plane (RHP). The initial value of the static gain of the Maxwell model $K_{0}=0.605$, so even a moderate increase of the gain from 0.605 to 1.41 can lead to instability and the same is likely to be true for real valve/WDN systems.

\section{NETWORK MODEL}

\section{Model schematic}

In order to simplify our theoretical static gain calculations presented in the next section as well as dynamic simulations used to recreate the real-life PRV instability event briefly described in the Introduction, the hydraulic model was reduced to just three components: the upstream pipe, the 
valve, and the downstream pipe. The rest of the network, shown in dotted lines in Fig. 8 was not modeled. Instead, it was assumed that the demands in all major nodes could be represented as pressure dependent outflow from the end of the downstream pipe and modeled with a modified Toricelli's orifice equation: $Q\left(c(t), H_{4}(t)\right)=c(t)\left(H_{4}(t)-z_{4}\right)^{\alpha}$ in which the pressure dependency coefficient $\alpha$ may differ from the original Toricelli's value of 0.5 (see Ferrante et al. (2014)). However it needs to be noted that the value of $\alpha$ in this study is likely to be different from the one in Ferrante et al. (2014) since we consider the pressure at the entry to a district metering area (DMA) whilst Ferrante et al. (2014) used the average pressure within the DMA. Since pressure changes at the nodes far down in the network constitute just a fraction of the changes in the DMA inlet pressure, the pressure dependency coefficient is likely to be lower from the ones reported by Ferrante et al. (2014), although this remains yet to be proven. In the equation above $z_{4}$ denotes the elevation of node $4, H_{4}$ is the pressure head at node 4 and $c(t)$ denotes the time-varying equivalent total orifice area at the end of the downstream pipe, i.e. DMA inlet (see Fig. 8).

The system is thus modeled as pressure-driven, not demand-driven. The demand-driven approach is not valid for dynamic systems because it reverses causality between flow and pressure. In demand-driven approach often used in steady-state calculations the flow is forced and the nodal pressures are calculated to produce the desired flows. In other words, changes in nodal pressure values are the effect of flow variations, whilst in physical systems it is the flow which is driven by pressure difference. Whilst such reversal of causality allows us to predict steady-state nodal pressures in a network under given demands, such an approach is not valid in dynamic simulation in which dynamic effects of inertia and water compressibility must not be neglected. In particular, demand-driven approach applied to dynamic system simulation neglects energy dissipation through openings (leaks and orifices at demand nodes) which occurs when the incoming pressure forces excess water outside of the system. This leads to a higher buildup of potential energy inside the system, resulting in overestimation of pressure wave amplitudes - see Jung et al. (2009) for comparison of both approaches on larger network models. 


\section{Model equations}

\section{Algebraic model}

The algebraic steady-state model of the system depicted in Fig. 8 is composed of four algebraic equations representing pressure drops across all three components of the network: the upstream pipe, the PRV, and the downstream pipe, supplemented with flow continuity equation. The resulting system of equations can be written as $\mathbf{F}(x, \mathbf{y})=\mathbf{0}$ where

$$
\mathbf{F}(x, \mathbf{y})=\left\{\begin{array}{l}
H_{\text {in }}-H_{u}-R_{1} Q^{2} \\
H_{u}-H_{d}-\frac{1}{K_{v}(x)} Q^{2} \\
H_{d}-H_{\text {out }}-R_{2} Q^{2} \\
Q-c\left(H_{\text {out }}-z_{4}\right)^{\alpha}
\end{array}\right.
$$

in which $x$ denotes the valve position and $\mathbf{y}=\left(H_{u} H_{d} H_{\text {out }} Q\right)^{T}$.

\section{Dynamic model}

The dynamic simulation model of the upstream and downstream pipe is composed of two water-hammer equations solved with the well-known method of characteristics (Wylie and Streeter 1993) which transforms the two partial differential water-hammer equations into two ODEs which are solved for each internal point along the length of the pipe, i.e. $\forall i \in\{2, . ., n-1\}$, where $n$ is the number of nodes in the conduit. In a short form these two ODEs can be written as two characteristic equations: $C^{+}: H_{i}=C_{p, i}-B Q_{i}$ and $C^{-}: H_{i}=C_{m, i}+B Q_{i}$. where $C_{p, i}=H_{i-1}^{*}+B Q_{i-1}^{*}-R Q_{i-1}^{*}\left|Q_{i-1}^{*}\right|$ and $C_{m, i}=H_{i+1}^{*}-B Q_{i+1}^{*}+R q_{i+1}^{*}\left|q_{i+1}^{*}\right|$ in which $B=\frac{a}{g A}$ and $R=\frac{\lambda \Delta x}{2 g D A^{2}}, \Delta x$ is the distance between internal pipe nodes and * denotes the value of the variable recorded in the previous time step. The initial flow value $Q_{0}$ in the system and the piezometric head values at points 2,3 , and 4 (see Fig. 8) required for the solution of the water-hammer model are obtained from Eq. 5 for $c(t=0)=c_{0}$ and $x(t=0)=x_{0}$. All internal node flow values within the pipes are assigned the initial flow value $Q_{0}$, i.e. $\forall i \in\{1, . ., n\}: Q_{i}=Q_{0}$. The internal head values $H_{i}$ are obtained through a linear interpolation between the boundary head values. The boundary 
conditions for the water-hammer model are listed in Table 1. Both pipes combined are discretized into $n-1$ segments, in which node $m$ represents point 2 , node $m+1=$ point 3 , whilst node $n$ represents point 4 in Fig. 8.

\section{CALCULATION OF THE STATIC GAIN OF THE VALVE/WDN SYSTEM}

It is argued in this paper that the static valve/WDN gain strongly depends on valve position, i.e. $K=K(x)$. The gain is low for large valve opening and increases gradually as the valve opening is reduced. The increase in the value of the gain as the valve position changes from $x=80 \%$ to $x=30 \%$ can be as high as 5 fold which presents a challenge for designing an effective controller. Since valve position influences the flow, the plant gain is also dependent on flow, i.e. $K=K(Q(x))$. As shall be shown below, for a PRV-controlled DMA under a constant $H_{d}$ we can determine a unique relationship between $x$ and $Q$ and since $Q$ increases as the valve is opened, $K$ is high for low flows making the valve less stable under low-flow conditions and supporting the generally accepted opinion that PRVs become unstable under small flows.

From definition $K(x)=d H_{d} / d x$ and can be calculated from the system of equations given in Eq. 5 using the implicit function theorem (see e.g. Hubbard and Hubbard 2015) which states that if we have an implicit system of nonlinear equations $\mathbf{F}(x, \mathbf{y})=\mathbf{0}$, such as one given in Eq. 5 , and it satisfies some mild conditions on its partial derivatives, then we can calculate the vector of derivatives: $\frac{d \mathbf{y}}{d x}=-\mathbf{F}_{y}{ }^{-1} \mathbf{F}_{x}$ where $\mathbf{F}_{x}$ represents the vector of derivatives of $\mathbf{F}$ with respect to $x$ and $\mathbf{F}_{y}$ represents the matrix of derivatives of $\mathbf{F}$ with respect to $\mathbf{y}$. Through differentiation of our system of equations given in Eq. 5 we obtain:

$$
\mathbf{F}_{x}=\left(\begin{array}{c}
0 \\
2 \frac{1}{\left[K_{v}(x)\right]^{3}} \frac{d K_{v}}{d x} Q^{2} \\
0 \\
0
\end{array}\right)
$$




$$
\mathbf{F}_{y}=\left(\begin{array}{cccc}
-1 & 0 & 0 & -2 R_{1} Q \\
1 & -1 & 0 & -2 \frac{1}{\left[K_{v}(x)\right]^{2}} Q \\
0 & 1 & -1 & -2 R_{2} Q \\
0 & 0 & -\alpha c\left(H_{\text {out }}-z_{4}\right)^{\alpha-1} & 1
\end{array}\right)
$$

where $\mathbf{y}=\left(H_{u} H_{d} H_{\text {out }} Q\right)^{T}$. By visual inspection we can see that the columns of $\mathbf{F}_{y}$ are linearly independent and thus $\mathbf{F}_{y}$ is invertible with a non-zero determinant:

$$
\operatorname{det} \mathbf{F}_{y}=-\left[1+2 \alpha c\left(H_{\text {out }}-z_{4}\right)^{\alpha-1} Q\left(R_{1}+R_{2}+\frac{1}{\left[K_{v}(x)\right]^{2}}\right)\right]
$$

We can also see that $\mathbf{F}_{x}$ has only one non-zero element $\mathbf{F}_{x}(2)$ and thus in order to calculate $d H_{d} / d x$ we only require to determine $\mathbf{F}_{y}^{-1}(2,2)$. Then

$$
\frac{d H_{d}}{d x}=\frac{d \mathbf{y}(2)}{d x}=-\mathbf{F}_{y}^{-1}(2,2) \cdot \mathbf{F}_{x}(2)
$$

After applying a co-factor expansion with respect to row 2 and column 2 we obtain the following relationship for $\mathbf{F}_{y}^{-1}(2,2)$

$$
\mathbf{F}_{y}^{-1}(2,2)=\frac{1+2 R_{2} Q \alpha c\left(H_{\text {out }}-z_{4}\right)^{\alpha-1}}{\operatorname{det} \mathbf{F}_{y}}
$$

which after substitution into Eq. 9 yields the following equation for the static gain of the valve/WDN system displayed in Fig. 8

$$
\frac{d H_{d}}{d x}=\frac{1+2 R_{2} Q \alpha c\left(H_{\text {out }}-z_{4}\right)^{\alpha-1}}{1+2 \alpha c\left(H_{\text {out }}-z_{4}\right)^{\alpha-1} Q\left(R_{1}+R_{2}+\frac{1}{\left[K_{v}(x)\right]^{2}}\right)} \cdot 2 \frac{1}{\left[K_{v}(x)\right]^{3}} \frac{d K_{v}}{d x} Q^{2}
$$

where $Q=c\left(H_{\text {out }}-z_{4}\right)^{\alpha}$

\section{Static gain of an isolated PRV}

Before we begin to analyze Eq. 11 let us first look at a simple case of an isolated PRV where $Q=$ const and $H_{u}=$ const and calculate the static gain of the PRV in this isolated scenario in which 
the PRV has no interaction with the rest of the network. We can accomplish this task two-fold. First, we can investigate a single valve equation, i.e. the second equation in Eq. 5

$$
H_{u}-H_{d}-\frac{Q^{2}}{\left[K_{v}(x)\right]^{2}}=0
$$

which can be rearranged to yield an explicit relationship for downstream head, $H_{d}=H_{u}-Q^{2} / K_{v}^{2}$. Differentiation of $H_{d}$ vs. $x$ under the assumption that $d H_{u} / d x \equiv 0$ and $d Q / d x \equiv 0$ yields

$$
\frac{d H_{d}}{d x}=\frac{d}{d x}\left(H_{u}-\frac{Q^{2}}{\left[K_{v}(x)\right]^{2}}\right)=2 Q^{2} \frac{1}{\left[K_{v}(x)\right]^{3}} \frac{d K_{v}(x)}{d x}
$$

which shows that the valve gain is inversely proportional to the valve capacity $K_{v}$ in third power. Although the term $d K_{v} / d x$ has some influence on the value of the valve gain, $d H_{d} / d x$ is most sensitive to $K_{v}^{3}$, which shows that the change in the valve gain between low and high valve openings (e.g. nonlinearity in the control system) is not so much a result of a hydraulic nonlinearity in the valve but the fact that the valve capacity itself is low under low openings. We can also see that the isolated PRV gain described by Eq. 13 is equal to the second factor in the connected valve/WDN gain given by Eq. 11 demonstrating that the static gain of the valve/WDN system is a product of interaction between the static gain of the isolated PRV and the hydraulic characteristics of the WDN, specifically pipe resistances/conductivities and the pressure dependency coefficient of the demand(s).

Another way of looking at the isolated PRV gain is through the elements of the matrix $\mathbf{F}_{y}$. Under assumption that $Q=$ const the elements in rows 1,2 and 3 in column 4 of $\mathbf{F}_{y}$ become null and $\mathbf{F}_{y}$ turns into a lower triangular matrix for which the determinant is a multiplication of all elements along the leading diagonal (1 in our case) and $\mathbf{F}_{y}^{-1}(2,2)=1 / \mathbf{F}_{y}(2,2)=-1$. Hence, $d H_{d} / d x=\mathbf{F}_{x}(2)$ and satisfies Eq. 13.

\section{Gain of the PRV connected to the network}

Pressure and flow in the valve/WDN system is a result of an interaction between the valve (valve characteristic) and the water distribution network (system curve). The theoretical system 
curve depends on the assumed demand model, namely its pressure dependency characteristics. In the extreme case where demands are assumed to be forced and independent from nodal pressures the system curve $Q\left(H_{d}\right)$ is constant and equal to the total demand in the system. In the general case with demands depending on pressures the total flow $Q$ through the PRV will increase with the downstream head, $H_{d}$. In such a case, i.e. with pressure-dependent demands, we can see that the static gain of the isolated PRV described by Eq. 13 and the second term in Eq. 11 is scaled by the first term in Eq. 9 which considers the properties of the WDN, i.e. pipe resistances, valve capacity and pressure dependency characteristics.

As shall be shown in numbers in the next section, this scaling function is always lower than unity which means that the interaction between the network and the valve leads to the reduction of the static valve/WDN gain compared to the isolated PRV gain. This behavior is very intuitive and can be easily explained. Changing valve position $x$ leads to the change in the energy loss across the valve which in turn alters the total (potential + kinetic) energy in the system. In the isolated case, or where demands are forced, i.e. $Q=$ const the kinetic energy remains constant and hence variation in $x$ leads to the change in potential energy only, i.e. nodal pressures. In the scenario with PRV connected to WDN and with pressure dependent demands both potential energy (nodal pressures) and kinetic energy (flow) are affected by the changes in $x$ and thus the variation in nodal pressures will be lower as some part of the total energy is being diverted into kinetic energy. Therefore, $d H_{d} / d x$ will always be lower in the valve/WDN system with pressure dependent demands compared to the isolated PRV scenario. The relationship between the closed-loop gain of the PRV connected to the network and the gain of the isolated PRV can be written as:

$$
K_{\text {connected }}(x)=f(\mathbf{p}) K_{\text {isolated }}(x)
$$

where $\mathbf{p}$ is a vector of parameters characterizing the hydraulic properties of the network, the valve, and pressure-dependency of the demands. If we look at the first term in Eq. 11 we can see that $f(\mathbf{p})<1$ since $R_{1}, R_{2}$ and $K_{v}$ are always greater than zero. 


\section{CASE STUDY}

This manuscript began with a brief description of a real-life instability event which occurred in a large-scale pressure control scheme installed in a WDN of one of the major cities in the UK. This instability event shall be used here as a source of data for the case-study which aim is to test the validity of the theoretical work presented above and to showcase via simulation the applicability of the proposed remedy against instabilities for electronically controlled PRVs.

The instability event under study is shown in Fig. 2 consisting of two subplots. The top subplot shows the outlet pressure $p_{\text {out }}$ from the PRV whilst the bottom subplot shows the valve position $x$. The pressure set-point set at 0.65 bar is marked with a thick dashed line and the \pm 0.1 bar dead zone between which the valve element is not actuated is shown with two horizontal thick solid lines. In both subplots the time scale corresponds to the morning hours of the day where the instability event occurred. The instability began around 5.37 a.m. with relatively small pressure oscillations between 0.25 bar and 1.0 bar which then went completely out of control around 6:24 a.m. when the valve opening started to oscillate between the minimum and the maximum allowed values of $0 \%$ and $80 \%$ respectively resulting in downstream pressure variations between 0 and 4 bars. Around 6:45 a.m. the amplitude of pressure oscillations began to decrease as a result of increasing flow through the valve. Although the flow is not displayed in the plots, it can be inferred from the valve position. As shall be shown later in Fig. 10 a direct positive and mildly nonlinear relationship exists between flow and valve position in the PRV-controlled system under study and this relationship is likely to be similar for other valve/WDN systems operating under the constant outlet pressure setpoint. Around 8:20 a.m. the oscillations decayed and the system resumed normal operation and began to keep the outlet pressure at the setpoint value of $0.65 \pm 0.1$ bar. This return to stability coincided with an increase in flow rate through the valve.

The PRV under study is fed by a fixed head reservoir with static pressure head $H_{\text {in }}=186.5$ $\mathrm{m}$ connected to the PRV through $\sim 10 \mathrm{~km}$ pipe of $0.8 \mathrm{~m}$ diameter. The PRV is set to drop the upstream pressure to $106.5 \mathrm{~m}$ which, considering pressure losses in the upstream pipe, results in an average pressure head drop of about $75 \mathrm{~m}$. The downstream pipe goes for a distance of about 2 
$\mathrm{km}$ after which it starts dividing into a dense network of pipes feeding different DMAs in the city. The PRV is controlled electronically by a PLC implementing a time-discrete proportional integral derivative controller (PID).

The work presented in this section proceeds as follows. First, pipe resistances of the simplified static pipe-PRV-pipe model from Fig. 8 are calibrated, given the PRV characteristic obtained from the measurements, such that the static hydraulic characteristic of the model matches that of the real network. This calibrated simplified static model is then used to derive the static gain curves of the valve/WDN system and to calculate the formula for the gain compensator which aim is to keep the static gain of the system constant within the entire permissible valve opening range. The positive effects of the gain compensator on the valve stability are then tested via dynamic simulation.

\section{Valve capacity curve}

The valve capacity curve was provided by the manufacturer and additionally calculated from 15min measurements of flow, upstream and downstream pressures, and valve position. As pictured in Fig. 9 the manufacturer's capacity curve and the measured capacity curve are significantly different indicating that either the valve was not made to the specification or, more likely, that the valve's capacity had changed over time due to wear and tear of internal components. The manufacturer's capacity curve and the capacity curve from the measurements were obtained via nonlinear curve fitting and are plotted in Fig. 9 with a thick solid and thick dash-dotted line, respectively. The obtained capacity curve equations are as follows. The manufacturer's valve capacity curve: $K_{v, \text { manu }}=0.2360 x^{2}+0.2491 x$, the valve capacity curve from the measurements: $K_{v \text {, meas }}=0.1597 x^{2}-0.01129 x$ The shaded surface between both curves represents the valve capacity uncertainty region indicating that the change of valve capacity over time needs to be accounted for at a controller design stage.

\section{Relationship between valve position and flow}

Data points obtained from 15-min measurements of flow and valve position and plotted in Fig. 10 as gray dots show that for our valve/WDN system a direct and slightly nonlinear relationship exists between valve position and flow. This relationship was found to be quadratic with the line of best fit 
given as $Q(x)=0.1155 x^{2}+0.3458 x$ which is marked in Fig. 10 with a thin dashed line. From the control point of view the existence of such a relationship allows us to infer the flow rate from the valve position or vice versa. This additional information can be used as a surrogate measurement for control purposes or sensor fault diagnosis. In this paper, valve position and flow measurements obtained from the case study were used to calibrate the static hydraulic properties of our simplified pipe-PRV-pipe model shown in Fig. 8 such that the model and the real network exhibit similar hydraulic characteristics. For this purpose we used the valve capacity curve obtained from the measurements, as explained in the section above. Our simplified model is governed by the system of equations given in Eq. 5. As we can see, the parameters which can be used to calibrate the hydraulic characteristics are the upstream and downstream pipe resistances $R_{1}, R_{2}$ and the pressure dependency coefficient $\alpha$. During calibration it was found that $\alpha$ was not a sensitive parameter. Hence, for simplicity, we assumed $\alpha=0.5$, as in the Toricelli's equation.

Since the pipe resistance coefficients, i.e. $R_{1}$ and $R_{2}$ lump three parameters characterizing the pipes' hydraulics, i.e. length $L$, absolute roughness $\epsilon$ and diameter $D$ we cannot uniquely find the right combination of these three parameters in this study. They are nevertheless very important in dynamic simulations as they affect in different ways how the pressure builds up and propagates across the network. The product of $L$ and $D^{2}$ (multiplied by $\pi / 4$ ) determines the total volume of water in the pipes and thus the amount of inertia in the system. The higher the inertia the higher the amplitude of pressure waves produced during valve closure/opening as the forces acting on the closing element are proportional to the accelerating/decelerating mass. The pipe length $L$ determines the period of pressure waves departing from/arriving at the PRV for a given wave speed. The absolute pipe roughness $\epsilon$ determines the amount of dissipative friction forces in the system and affects the pressure drop in the pipe in steady-state as well as the amount of pressure wave attenuation during transient flow. Bearing this in mind the calibrated parameters $R_{1}$ and $R_{2}$ were first adjusted such that the model characteristics matched the measurements. Then the information about pipe friction coefficients and upstream and downstream pipe diameters was taken from site, allowing the authors to adjust the pipe lengths $L_{1}$ and $L_{2}$. In case some of the above information is 
not available and need to be assumed the results of the transient simulations may differ significantly from the measurements in terms of pressure wave period, pressure wave amplitudes and amplitude attenuation. These discrepancies may be reduced during further calibration on a dynamic model by adjusting, specifically, the values of pipe lengths, pipe diameters, and pipe roughness coefficients.

As mentioned above, the pressure dependency coefficient $\alpha$ was found to be an insensitive parameter. The verbal explanation of this fact is as follows. An increase in $\alpha$ will produce a higher flow for a given value of pressure head $\mathrm{H}_{4}$ or orifice opening $c$ (see Fig. 8). If the flow increases and $H_{3}$ is kept constant, i.e. by the PRV, then the valve needs to open in order to reduce the pressure drop which is proportional to $Q^{2}$. Hence $Q$ and $x$ will increase/decrease in the same direction and the $Q$ vs. $x$ curve will not change by much. Nevertheless, we should bear in mind that the value of $\alpha$ has a significant impact on the transient flow as it determines how much potential energy entering with the wave as pressure is lost in the system as the water is ejected through the orifices (openings) at individual nodes or, in our case, at the end of the downstream pipe.

The effects of $R_{1}$ and $R_{2}$ on the $Q$ vs $x$ characteristic of our simplified hydraulic model are as follows. If $R_{1}$ is increased then the pressure drop in the upstream pipe will be more sensitive to flow, thus $H_{2}$ will decrease more with respect to $Q$ and hence we will need to have a higher valve opening $x$ for a given flow, since the pressure drop across the PRV will need to be smaller to maintain constant downstream pressure. The curve will move left if $R_{1}$ is increased. If $R_{2}$ is increased then the pressure drop in the downstream pipe will be more sensitive to flow, thus $H_{4}$ will be lower for a given flow and the flow, since it's pressure dependent, will decrease. Hence, we will have lower flows for the same opening. The curve will move down if $R_{2}$ is increased.

The calibration was performed manually. The obtained model properties are as follows: $L_{1}=$ $5,000 \mathrm{~m}, L_{2}=10,000 \mathrm{~m}, D_{1}=D_{2}=0.8 \mathrm{~m}, \epsilon_{1}=\epsilon_{2}=0.003 \mathrm{~m}, z_{4}=50 \mathrm{~m}$. The calibrated curve is shown in Fig. 10 with a thick dash-dotted line. As we can see, the curve matches the measurements and is closely aligned with the line of best fit (thin dashed line). Additionally, we also plotted the curve for $K_{v}=K_{v, \text { manu }}$, i.e. for the manufacturer's valve capacity characteristic. Since the manufacturer's valve capacity is higher from the measured one the system would allow 
higher flows for a given valve opening and therefore the curve (thick solid line) lies above the measurements.

\section{Valve gain analysis}

The calibrated parameter values and the measured valve characteristic were fed into Eq. 11 and used for subsequent calculation of the static valve/WDN gain vs. valve opening for different values of $\alpha$. Additionally, Eq. 13 was used to calculate the isolated PRV gain, i.e. for $Q=$ const and $H_{u}=$ const. The resulting curves were plotted in Fig. 11. As predicted, the static valve/WDN gain decreases with valve opening. The difference in gain between low and high valve openings is most severe for the isolated PRV case and becomes smaller in the connected valve/WDN case due to the reducing effects of the WDN, i.e. dependency of flow on pressure. This interaction between the PRV and the rest of the network was already explained earlier on in the text - see Eq. 14. Fig. 11 shows that the gain vs. valve position curve becomes less steep as $\alpha$ increases. Whilst the gain can change from 1 at $x=80 \%$ to 6 at $x=20 \%$ for $\alpha=0.5$, this change can be as severe as 1 to 10 for $\alpha=0.3$.

The gain values were calculated for two valve characteristics: the manufacturer's characteristic $K_{v, \text { manu }}\left(\right.$ shown in solid line) and the measured characteristic $K_{v, \text { meas }}$ (shown in dashed line). As can be seen, both curves lie close together for medium valve openings between $30 \%$ and $60 \%$ and began to diverge at extreme values close to the minimum and maximum allowed openings of $20 \%$ and $80 \%$ respectively. The valve capacity has a larger impact on the $Q$ vs. $x$ characteristic (see Fig. 10) which means that the flows will be different for the same valve openings. Specifically, given that both PRV gain characteristics are similar and the gain is such that the valve begins to oscillate at around $30 \%$, the valve with the manufacturer's characteristic (e.g. brand new valve) will begin to oscillate at a higher flow than the valve with the measured characteristic (e.g. old valve).

The gain curve for $K_{v}=K_{v, \text { meas }}$ and $\alpha=0.5$ was approximated with a third order polynomial using nonlinear curve fitting. The obtained gain equation is given below.

$$
K_{\text {connected }}(x)=K(x)=-1.201 \times 10^{-5} x^{3}+3.162 \times 10^{-3} x^{2}-0.3186 x+12.23
$$


Eq. 15 shall be later used to formulate a nonlinear gain compensator used to prevent PRV instability under low flows conditions.

Fig. 12 shows the impact of our simplified network model parameters, i.e. $R_{1}, R_{2}$ and the valve characteristic $K_{v}(x)$ on the scaling function $f(\mathbf{p})$ featured in Eq. 14. For this purpose the first term of Eq. 11 representing $f(\mathbf{p})$ was split into three different individual terms: $f_{R_{1}}=2 R_{1} Q d Q / d \alpha$, $f_{R_{2}}=2 R_{2} Q d Q / d \alpha, f_{K_{v}}=2\left(1 / K_{v}(x)\right)^{2} Q d Q / d \alpha$ where $d Q / d \alpha=\alpha c\left(H_{\text {out }}-z_{4}\right)^{\alpha-1}$. Using these three new terms $f(\mathbf{p})$ can now be represented as:

$$
f(\mathbf{p})=f\left(R_{1}, R_{2}, K_{v}(x), \alpha\right)=\frac{1+f_{R_{2}}}{1+f_{R_{1}}+f_{R_{2}}+f_{K_{v}}}
$$

$f_{R_{1}}, f_{R_{2}}$ and $f_{K_{v}}$ were plotted in Fig. 12 for $\alpha=0.3$ and $\alpha=0.5$. As shown, all three terms show a stronger relationship vs. $x$ for higher $\alpha$ values. We can also see that the most dominant parameter is $f_{K_{v}}$, although the scaling effects of $R_{1}$ and $R_{2}$ become significant at higher openings. What this means is that if, i.e. $R_{1}$ or $R_{2}$ are increased the gain value at lower openings will remain similar whilst the gain reduction at larger openings shall be higher. The overall scaling factor $f$ for $\alpha=0.3$ and $\alpha=0.5$ was plotted in thick solid and thick dashed line respectively. As explained earlier using Eq. 14 the scaling factor is always less than unity.

\section{Control loop structure}

The valve/WDN control loop structure (see Fig. 13) and controller parameters are as follows. The valve with a known capacity characteristic described above is controlled with a time discrete PID with the following gains: $K_{p}=0.5 \% / \mathrm{m}, K_{i}=0.05 \% /(\mathrm{m} \mathrm{s})\left(\right.$ i.e. $\left.T_{i}=10 \mathrm{~s}\right), K_{d}=0(\mathrm{~m} \mathrm{~s}) / \%$; and with sampling time $t_{s}=0.1 \mathrm{~s}$. The actuator is modeled as a first order system with time constant of $0.1 \mathrm{~s}$. The valve opening and closing rates are limited at $\pm 100 / 87 \% / \mathrm{s}$ to reduce the risk of transients. The rate-limited actuator output then goes through a backlash block with a deadband of $0.8 \%$. The rate limiter and the deadband are embedded within the Actuator block and are not shown in the block diagram. The controller error $e$ is the difference between the downstream pressure head setpoint $H_{d}^{\text {set }}=106.5 \mathrm{~m}$ and the downstream pressure measurement $H_{d, \text { ave }}^{\text {meas }}(\mathrm{m})$ averaged using 
a moving average filter with a buffer size of 300 data points updated every $0.02 \mathrm{~s}$ and followed by a zero-order-hold of $0.1 \mathrm{~s}$. To reduce the amount of control effort the control system is only active when $|e| \geq 0.5 \mathrm{~m}$, i.e. the outlet pressure head $H_{d}$ is allowed to vary $\pm 0.5 \mathrm{~m}$ - see Dead zone block in Fig. 13.

\section{Nonlinear compensator for stability improvement}

One way to combat the nonlinearity in the valve/WDN system resulting from the dependence of static gain on valve position is to introduce a nonlinear compensator in the forward path of the feedback system which will scale the control signal depending on the current measurement of the valve position, such that the gain is constant (or near constant in practice) over the entire permitted range of valve openings. Placement of the nonlinear compensator in the feedback system structure is indicated in Fig. 13 with a dashed rectangle. The compensator receives the valve position measurement from the valve actuator and calculates the correcting factor either from a look-up table or from an analytical formula, such as the one derived below for our system. It is recommended that the PID is tuned at the typical valve position $x_{t y p}$ around $40 \%-50 \%$. The correcting factor can then be calculated from the following formula.

$$
k(x)=\frac{K_{p}\left(x=x_{\text {typ }}\right)}{K_{p}\left(x_{\text {meas }}\right)}
$$

where $x$ is the actual valve position, $x_{\text {meas }}$ is the measured valve position and $x_{t y p}$ is the typical valve position for which the controller was tuned. If during operation of the control system the valve position changes from $x_{t y p}$ to $x$, the gain, as seen by the controller, will theoretically remain the same as for $x=x_{t y p}$.

$$
K=K(x) k(x)=K(x) \frac{K\left(x=x_{\text {typ }}\right)}{K\left(x_{\text {meas }}\right)} \approx K\left(x=x_{\text {typ }}\right)
$$

The above equation is valid if $x_{\text {meas }} \approx x$, i.e. we have an accurate measurement of valve position and we have a perfect compensation model $k(x)$ under all operating conditions. In practice, $K \neq$ const since $x \neq x_{\text {meas }}$ and the compensator model will never be perfect. However, in theory, assuming 
perfect valve position measurements and perfect gain compensation the PID gains, i.e. $K_{p}, K_{i}$ and $K_{d}$ tuned for the typical valve position should, in theory, be valid and satisfying the control system design criteria across the entire operating range of the PRV. The static gain correction formula was obtained from Eq. 17 assuming the model hydraulic structure in Fig. 8, $\alpha=0.5, K_{v}=K_{v, \text { meas }}$ and the typical valve position $x_{t y p}=50 \%$ for which the fitted polynomial curve (see Fig. 11) was calculated to be:

$$
k(x)=\frac{K\left(x_{\text {typ }}=50 \%\right)}{K(x)}=\frac{2.340}{-8.280 \times 10^{-6} x^{3}+2.450 \times 10^{-3} x^{2}-0.2658 x+10.54}
$$

$k(x)$ curve was plotted in Fig. 14. The plot shows that the scaling function $k(x) \equiv 1$ for $x=x_{t y p}$, in our case $x=50 \%$ whilst $k(x)<1$ for $x<x_{t y p}$ and $k(x)>1$ for $x>x_{\text {typ }}$, thus maintaining the plant gain at a constant value equal to $K\left(x=x_{t y p}\right)$.

\section{Valve/WDN simulation with and without static gain compensator}

The purpose of the transient simulation described in this section is to recreate the instability event visualized in Fig. 2, and to show the improvement in the stability of the control system after the introduction of the static gain compensator. We aim to demonstrate the validity of the theoretical concepts introduced earlier in this manuscript and derived via static analysis by testing them in a transient simulation scenario with control, actuation and sensing loop taken from the real-life case study. Although the transient model was not properly calibrated on dynamic data we shall see that the simulation results and the actual instability event are indeed very similar. The second aim of this simulation study was to demonstrate that instabilities in valve/WDN systems can occur with zero disturbance, i.e. with smooth inputs, in order to reinforce the idea that the loss of stability in PRVs is mainly a result of changing static plant gain and is not in any way caused by transients, although an incoming pressure wave or change in demand can trigger the instability earlier if the valve/WDN system is already operating close to instability. A similar simulation study was already performed by Janus and Ulanicki (2017).

The simulations were performed on the simplified hydraulic model shown schematically in 
Fig. 8 using water-hammer equations solved with the method of characteristics, given the boundary conditions in Table 1 and assuming that the wave speed $a=1,200 \mathrm{~m} / \mathrm{s}$. The hydraulic parameters, i.e. $L_{1}, L_{2}, D_{1}, D_{2}, \epsilon_{1}$ and $\epsilon_{2}$ were calibrated earlier from the information provided in the measured $Q$ vs. $x$ curve given in Fig. 10 and from site information about pipe roughness and diameters. The model was created and simulated in Simulink ${ }^{\mathrm{TM}}$ and MATLAB $2015 \mathrm{a}^{\mathrm{TM}}$.

The control, actuation and sensing loop shown in Fig. 13 was modeled as follows. The deadzone was described with a User Specific Function block with code (abs (u)>dead_zone)*u where $u$ is the block input. The rate limit on the actuator is implemented as a Rate Limiter block whilst the actuator backlash is implemented using the Backlash block. The discrete PID controller was modeled using the PID Controller Block with Backward Euler integration method and minimum and maximum output limits at $10 \%$ and $80 \%$ respectively. The pipes were discretized such that an appropriate ratio between the selected time step $\Delta t=0.02 \mathrm{~s}$ and the spacial step $\Delta x$ was maintained allowing proper capture of the characteristics at internal pipe nodes given the wave speed $a$.

The instability, with no gain compensator, was recreated by reducing the open orifice area at the end of the downstream pipe in a linear fashion over $1 \mathrm{hr}$ from an initial value $A_{\text {init }}=1.3141 \times 10^{-2} \mathrm{~m}^{2}$ to the final value of $3.141 \times 10^{-3} \mathrm{~m}^{2}$, i.e. by $0.01 \mathrm{~m}^{2}$. A orifice was then held at the final value for the next 30 minutes after which the ramp reversed direction and the orifice area increased back to the initial value in the next hour. Hence, the total simulation time was equal to $2.5 \mathrm{hrs}$ but in order to better display the occurring oscillations the limits on the $\mathrm{x}$ axis in the simulation results plots (see Fig 15) were reduced to $0.5 \mathrm{~h}$ and $2.0 \mathrm{~h}$ respectively.

The top subplot in Fig. 15 shows that as the orifice area gradually got smaller so was the total flow $q_{P R V}$ in the network. In order to maintain a constant downstream pressure head $\left(\mathrm{H}_{3}\right)$ whilst the flow was being reduced the valve was closing in an almost linear fashion. In the scenario without the gain compensator (dark solid line) the pressure was within the allowed band for the first hour until the valve opening reached the value of about $30 \%$ under which the PRV lost stability and began to oscillate. The oscillations were rising in amplitude for the next half an hour whilst the open orifice area was kept at a constant and minimum value. The valve began to slowly regain stability, 
which manifested itself in a gradual reduction of the oscillation amplitude as $A_{\text {orifice }}$ was being increased and finally resumed normal operation at $t \sim 1.8 \mathrm{~h}$ when the valve opening increased to about $40 \%$. This simulated valve behavior is very similar to the observed real-life instability event (see Fig. 2) where the valve lost stability at $x=30 \%$ and regained stability at $x=60 \%$.

In the case with the static gain compensator in place (light solid line) we can see that the valve did not oscillate at low flow since the gain compensation formula lowered the static gain such that it didn't reach the critical vale for which the system becomes unstable. However, it has to be noted that this gain compensation task was achieved with a perfect compensator, i.e. without a mismatch between the simulation model and the model used to derive the compensator. Additionally, a very simple hydraulic model was used in the study which represented all distributed nodal demands by one time-varying orifice area. In real-life applications care must be taken that the uncertainty with regards to model structure and model parameters is taken into account during the design of a PRV controller or otherwise an appropriate robust field tuning method needs to be developed. Even though we had a perfect compensation model we can see in the middle subfigure in Fig. 15 that the gain compensated valve/WDN system also begins to oscillate, albeit very slightly, between 1 and 1.1 hrs. It is suspected that these small oscillations are due to a limit cycle produced by the nonlinearities in the control-loop, i.e. dead-zone, backlash, and $0.1 \mathrm{~s}$ zero-order-hold. It is suspected that the most significant nonlinearity at small openings is due to the backlash which, having a fixed value of $0.8 \%$, becomes proportionally large when the opening is small. Also, the $\pm 0.5 \mathrm{~m}$ dead-zone might have contributed to pressure variations, since it is a well-known fact that dead-zones in feedback control loops lead to limit cycles.

Based on the results of the above case study and the theoretical developments described in the first half of this manuscript we can formulate the following practical recommendations for a hydraulic engineer working in pressure management of WDNs. First, with all pressure control schemes incorporating a valve of any type, an engineer should be aware that the static gain between valve opening and outlet pressure can increase significantly as the valve opening is reduced, which in turn can lead to valve/WDN system instability under low flow conditions. The engineer should 
therefore first assess the gain at the nominal valve opening via theoretical calculation or from online measurements and later check the gain at the minimum flow conditions. If the difference between the nominal and the maximum gain is large, one should perform further analysis of system stability. The gain can be calculated from the valve characteristic curve combined with the network characteristics. However since the latter is difficult to obtain it is recommended that downstream pressure responses to step change e.g. $+0.5 \%$ in valve opening are recorded in openloop configuration, i.e. with disconnected feedback loop, at different flow rates. By taking the ratio between the pressure increase and the valve position change $\Delta H_{d} / \Delta x$ in steady-state we will calculate the static gain vs. valve position curve which can be used to design a gain compensator to be used in line with an electronic controller such that the overall static gain of the system remains at approximately constant value over an entire flow range. For hydraulic PRVs in which the controller is implemented in hardware it is necessary to introduce modifications to the pilot control loop in order to achieve similar compensation effects. This is the subject of the authors' current research. In cases where a modification to the control loop cannot be made, the risk of instability can be reduced by narrowing down the operating range of the control valve, such that the opening does not exceed the $30 \%-70 \%$ range. If due to high variability of flows the range of openings is high it may be possible to install a cascade of valves in which the upstream valve is a normal actuated valve with a time-scheduled opening trajectory following a diurnal demand pattern whilst the downstream PRV acts as a second step in pressure reduction whilst maintaining a constant outlet pressure.

\section{CONCLUSION}

This manuscript demonstrated that the static gain of a valve/WDN system decreases with valve opening, making outlet pressure more sensitive to valve position changes at low openings. This increase in valve gain at small valve openings and thus, flows, can alone explain why valves tend to become unstable under low-flow conditions, which was proved through simulation. The changing nature of the static valve/WDN gain is due to an inherent property of a valve whose capacity increases with valve opening, as well as hydraulic properties of the rest of the water distribution network. For simple networks an analytical relationship between the static valve/WDN gain and 
valve opening can be derived, whilst for larger networks an empirical procedure will need to be developed in order to be able to measure the static gain variability and then use this information to design countermeasures preventing the PRVs from oscillating. One such countermeasure, i.e. static gain compensator was tested here and proved to eliminate valve instability under low flow conditions. Such gain compensation is an easy solution for electronically controlled valves, however care must be taken that a robust solution to such compensation formula is sought in the future which is resilient to biased, delayed and noisy valve position measurements and to the mismatch between the network model and the real system. Similar compensation solution can be sought for hydraulically controlled PRVs, although the problem is likely to be more complicated as the control system there is realized mechanically which is harder to modify than programmable electronic controllers. It is envisaged that through appropriate alterations to control structures of both types of valves we can make them more stable and hence reduce the risk of occurrence of PRV-related and destructive transient effects in water distribution networks. It is possible that further improvements can be made through appropriate selection of the type of the control valve and its sizing in order to reduce the nonlinearity and narrow down the operating range of the valve hence reducing the difference between the minimum and the maximum static gain. However, the problem of varying static gain will still persist and will have to be accounted for during controller design, e.g. via static gain compensation.

\section{ACKNOWLEDGMENTS}

The authors wish to thank Alan Woodburn for his valuable comments and providing the data for the Case Study.

\section{NOTATION}


The following symbols are used in this paper:

$$
\begin{aligned}
A & =\text { pipe cross section area; } \\
A_{\text {orifice }} & =\text { orifice opening area; } \\
a & =\text { pressure wave speed; } \\
c & =\text { equivalent total orifice area at the end of the downstream pipe; } \\
D & =\text { pipe diameter; } \\
e & =\text { error signal; } \\
f & =\text { gain scaling factor; } \\
f_{R_{1}}, f_{R_{2}}, f_{K_{v}} & =\text { gain correcting factors due to upstream pipe, downstream pipe and valve capac } \\
G & =\text { transfer function; } \\
H & =\text { pressure head; }
\end{aligned}
$$

$H_{u}, H_{d}, H_{d}^{\text {set }}, H_{\text {out }}, H_{\text {in }} \quad=\quad$ upstream, downstream, downstream setpoint, outlet, and inlet pressure head;

$K=$ plant gain;

$K_{\text {connected }}, K_{\text {isolated }}=$ connected and isolated PRV gain

$K_{e}=$ fluid's bulk modulus of elasticity;

$K_{p}, K_{i}, K_{d}=$ proportional, integral, and derivative gains of a PID controller;

$K_{v}=$ valve capacity;

$K_{v, \text { manu }}, K_{v \text {,meas }}=$ valve capacity from manufacturer's data and measurements;

$k=$ gain compensation coefficient;

$L=$ pipe length;

$p_{\text {out }}=$ outlet pressure;

$Q=$ flow;

$R=$ pipe resistance;

$s=$ Laplace variable;

$T_{i}=$ PID controller's integral time constant;

$t=$ time;

$t_{d}=$ time delay;

$t_{s}=$ sampling time;

$u=$ control signal;

$x, x_{\text {typ }}, x_{\text {meas }}, x_{0}=$ valve position, typical valve position, measured valve position, initial valve po

$y=$ output;

$z=$ elevation;

$\alpha=$ pressure dependency coefficient;

$\epsilon=$ absolute pipe roughness;

$\lambda=$ Darcy-Weisbach friction coefficient;

$\omega_{n}=$ natural frequency;

$\rho=$ fluid density;

$\tau=$ time constant;

$\zeta=$ damping ratio; 


\section{REFERENCES}

Berardi, L., Laucelli, D., Ugarelli, R., and Giustolisi, O. (2015). “Leakage Management: Planning Remote Real Time Controlled Pressure Reduction in Oppegård Municipality.” Procedia Engineering, 119, 72-81.

Brunone, B. and Morelli, L. (1999). “Automatic Control Valve - Induced Transients in Operative Pipe System.” Journal of Hydraulic Engineering, 125(5), 534 - 542.

Campisano, A., Modica, C., Reitano, S., Ugarelli, R., and Bagherian, S. (2016). "Field-Oriented Methodology for Real-Time Pressure Control to Reduce Leakage in Water Distribution Networks." Journal of Water Resources Planning and Management, 142(12).

Campisano, A., Modica, C., and Vetrano, L. (2012). "Calibration of Proportional Controllers for the RTC of Pressures to Reduce Leakage in Water Distribution Networks." Journal of Water Resources Planning and Management, 138(4), 377-384.

Creaco, E., Campisano, A., Franchini, M., and Modica, C. (2017). "Unsteady Flow Modeling of Pressure Real-Time Control in Water Distribution Networks." Journal of Water Resources Planning and Management, 143(9).

Evans, W. R. (1950). “Control System Synthesis by Root Locus Method.” Transactions of the American Institute of Electrical Engineers, 69(1), 66-69.

Ferrante, M., Meniconi, S., and Brunone, B. (2014). "Local and global leak laws - the relationship between pressure and leakage for a single leak and for a district with leaks." Water Resources Management, 28(11), 3761 - 3782.

Ghorbanian, V., Karney, B. W., and Guo, Y. (2015). “The link between transient surges and minimum pressure criterion in water distribution systems." Pipelines, Baltimore, Maryland.

Giustolisi, O., Campisano, A., Ugarelli, R., Laucelli, D., and Berardi, L. (2015). "Leakage Management: WDNetXL Pressure Control Module.” Procedia Engineering, 119, 82-90.

Hazewinkel, M., ed. (1994). Encyclopaedia of Mathematics (set). Springer Netherlands, 1 edition. Hubbard, J. H. and Hubbard, B. B. (2015). Vector calculus, linear algebra, and differential forms: a unified approach. Matrix Editions. 
Janus, T. and Ulanicki, B. (2017). "Hydraulic modelling for pressure reducing valve controller design addressing disturbance rejection and stability properties." Procedia Engineering, 186, $635-642$.

Jung, B. B. S., Boulos, P. F., and Wood, D. J. (2009). "Effect of pressure-sensitive demand on surge analysis." American Water Works Association Journal.

Meniconi, S., Brunone, B., Ferrante, M., Mazzetti, E., Laucelli, D., and Borta, G. (2015). “Transient effects of self-adjustment of pressure reducing valves.” Procedia Engineering, 119(Supplement C), 1030 - 1038 Computing and Control for the Water Industry (CCWI2015) Sharing the best practice in water management.

Meniconi, S., Brunone, B., Mazzetti, E., Laucelli, D. B., and Borta, G. (2016). "Pressure Reducing Valve Characterization for Pipe System Management." Procedia Engineering, Vol. 162, 455-462.

Meniconi, S., Brunone, B., Mazzetti, E., Laucelli, D. B., and Borta, G. (2017). "Hydraulic characterization and transient response of pressure reducing valves: laboratory experiments." Journal of Hydroinformatics.

Ogata, K. (2010). Modern Control Engineering. Pearson, 5th edition.

Page, P. R., Abu-Mahfouz, A. M., and Yoyo, S. (2016). "Real-time Adjustment of Pressure to Demand in Water Distribution Systems: Parameter-less P-controller Algorithm.” Procedia Engineering, 154, 391-397.

Prescott, S. L. and Ulanicki, B. (2003). "Dynamic modeling of pressure reducing valves.” Journal of Hydraulic Engineering, 129(10), 804-812.

Thornton, J. and Lambert, A. (2005). "Progress in practical prediction of pressure: leakage, pressure: burst frequency and pressure: consumption relationships.” Proceedings of IWA Special Conference on Leakage, 12-14.

Ulanicki, B. and Skworcow, P. (2014). "Why PRVs tend to oscillate at low flows." Procedia Engineering, 89, 378-385.

Vicente, D. J., Garrote, L., Sánchez, R., and Santillán, D. (2015). "Pressure Management in Water Distribution Systems: Current Status, Proposals, and Future Trends.” Journal of Water Resources 
Planning and Management, 142(2), 04015061.

Wylie, E. B. and Streeter, V. L. (1993). Fluid Transients in Systems. Prentice-Hall; Facsimile edition (2 Jan. 1993). 
TABLE 1. Boundary conditions for the water-hammer model.

\begin{tabular}{ll}
\hline Upstream reservoir & PRV upstream \\
\hline$H_{1}=H_{\text {tank }}$ & $H_{m}=C_{p, m}-B_{1} Q_{m}$ \\
$Q_{1}=\frac{H_{\text {tank }}-C_{m, 1}}{B_{1}}$ & $Q_{m}=0.5 K_{v}^{2}\left(-\left(B_{1}+B_{2}\right)+\sqrt{\left(B_{1}+B_{2}\right)^{2}-4 K_{v}^{-2}\left(C_{m, m+1}-C_{p, m}\right)}\right)$ \\
\hline PRV downstream & Outlet orifice \\
\hline$H_{m+1}=C_{m, m+1}+B_{2} Q_{m+1}$ & $H_{n}=C_{p, n}-B_{2} Q_{n}$ \\
$Q_{m+1}=Q_{m}$ & $Q_{n}=g c^{2}\left(-B_{2}+\sqrt{B_{2}^{2}-\frac{2}{g c^{2}}\left(z_{n}-C_{p, n}\right)}\right)$ \\
\hline
\end{tabular}




\section{List of Figures}

1 Block diagram representation of a closed-loop feedback system including valve/WDN plant and a controller. . . . . . . . . . . . . . . . 35

2 PRV instability event recorded in a large-scale pressure control scheme in one of the major cities in the UK. . . . . . . . . . . . . . 36

3 Block diagram of the lumped inertial-oscillatory model of a valve/WDN system. . . 37

4 Schematic diagram of the physical Maxwell model. . . . . . . . . . . . . 38

5 Step response of the Maxwell model to a unit step in the input. . . . . . . . . . . 38

6 Step response of lumped inertial-oscillator model of a valve/WDN to a unit step in the input. . . . . . . . . . . . . . . . . . 39

$7 \quad$ Root-locus of the closed-loop system with the Maxwell model plus actuator inertia. 40

8 Schematic of the hydraulic model used for dynamic simulation and calculation of static gain. . . . . . . . . . . . . . . . . . . . . 41

9 Valve capacity according to the manufacturer's data and the measurements. Dashed lines denote $95 \%$ confidence intervals for the fitted curves. . . . . . . . . . . . 42

10 Relationship between total flow $Q$ and valve position $x$ for the distribution system under study and the simplified pipe-PRV-pipe model. . . . . . . . . . . . . . 43

11 Valve/WDN gain $K$ against valve position $x$ for different values of pressure dependency coefficient $\alpha \ldots \ldots \ldots \ldots \ldots$. . . . . . . . . . . . . . . . . . . . . .

12 Impact of $R_{1}, R_{2}$ and $K_{v}$ on the scaling function $f(\mathbf{p})$ between $K_{\text {connected }}(x)$ and

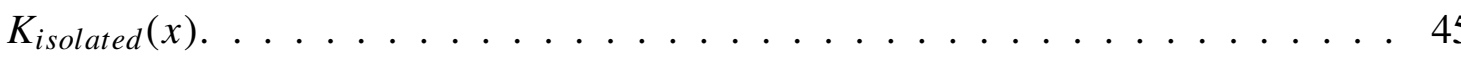

13 Valve/WDN closed-loop model structure adopted from real-life system and implemented in the mathematical model used for the transient simulation study. . . . . . 46

14 Nonlinear gain compensator curve $k(x)$ used for scaling the Valve/WDN plant gain $K$ in the transient simulation study. . . . . . . . . . . . . . . . . 47

15 Results of the transient simulations of our simplified pipe-PRV-pipe hydraulic model with (red) and without (black) gain compensation. . . . . . . . . . . . 48 


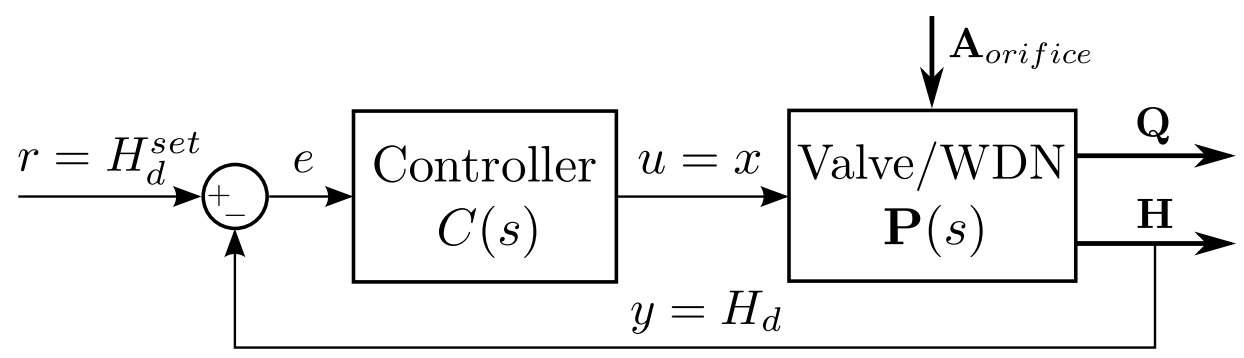

Fig. 1. Block diagram representation of a closed-loop feedback system including valve/WDN plant and a controller. 

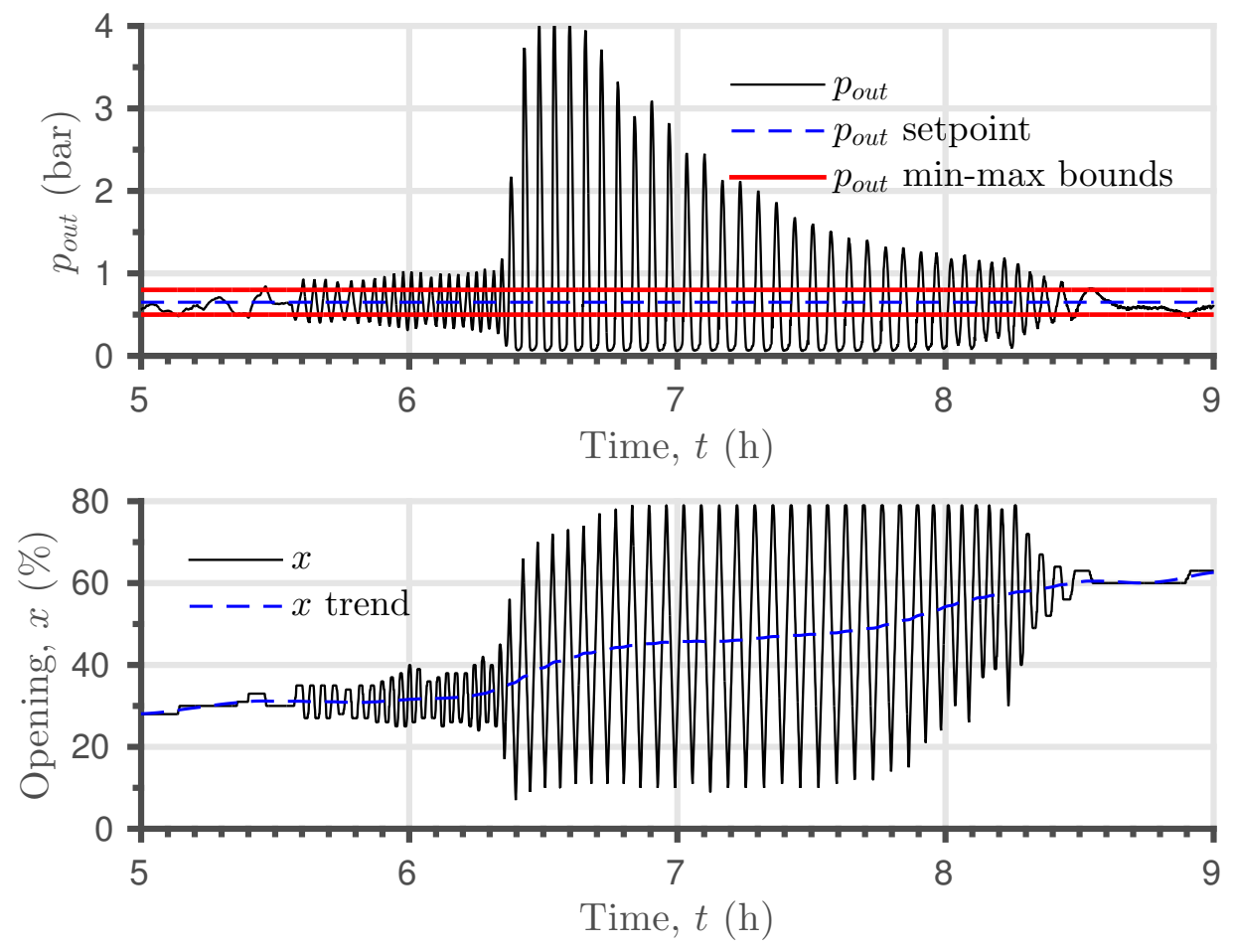

Fig. 2. PRV instability event recorded in a large-scale pressure control scheme in one of the major cities in the UK. 


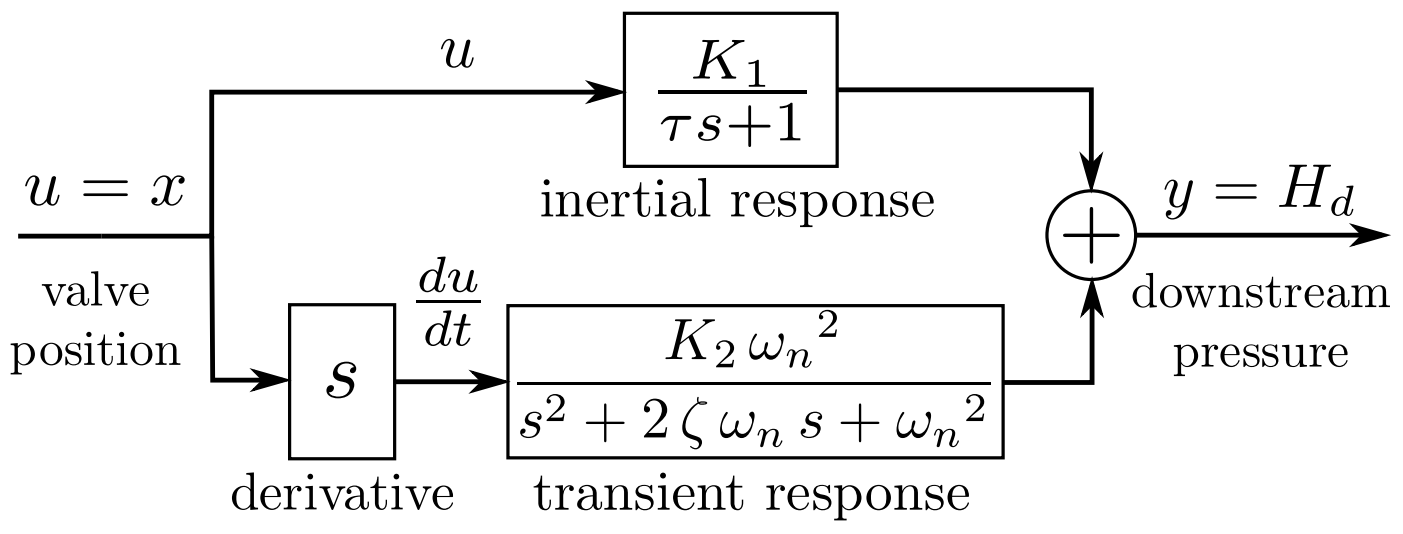

Fig. 3. Block diagram of the lumped inertial-oscillatory model of a valve/WDN system. 


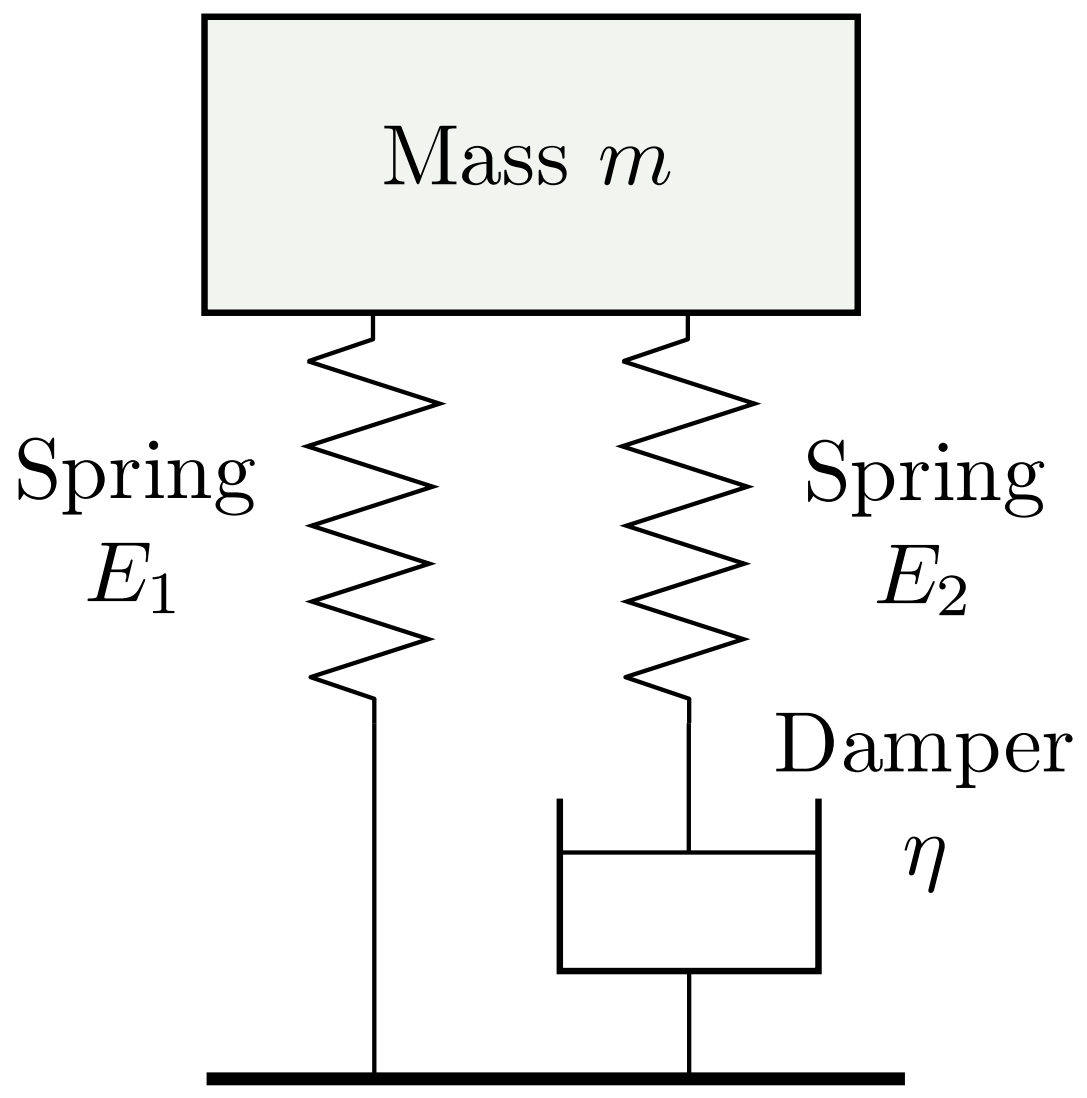

Fig. 4. Schematic diagram of the physical Maxwell model.

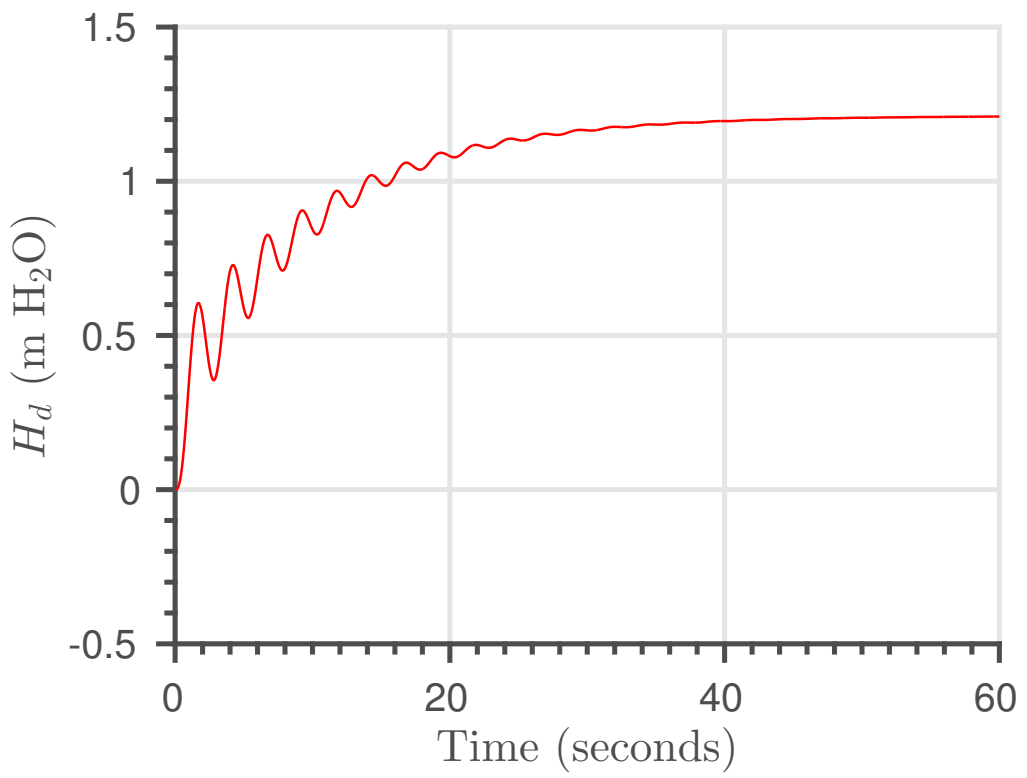

Fig. 5. Step response of the Maxwell model to a unit step in the input. 


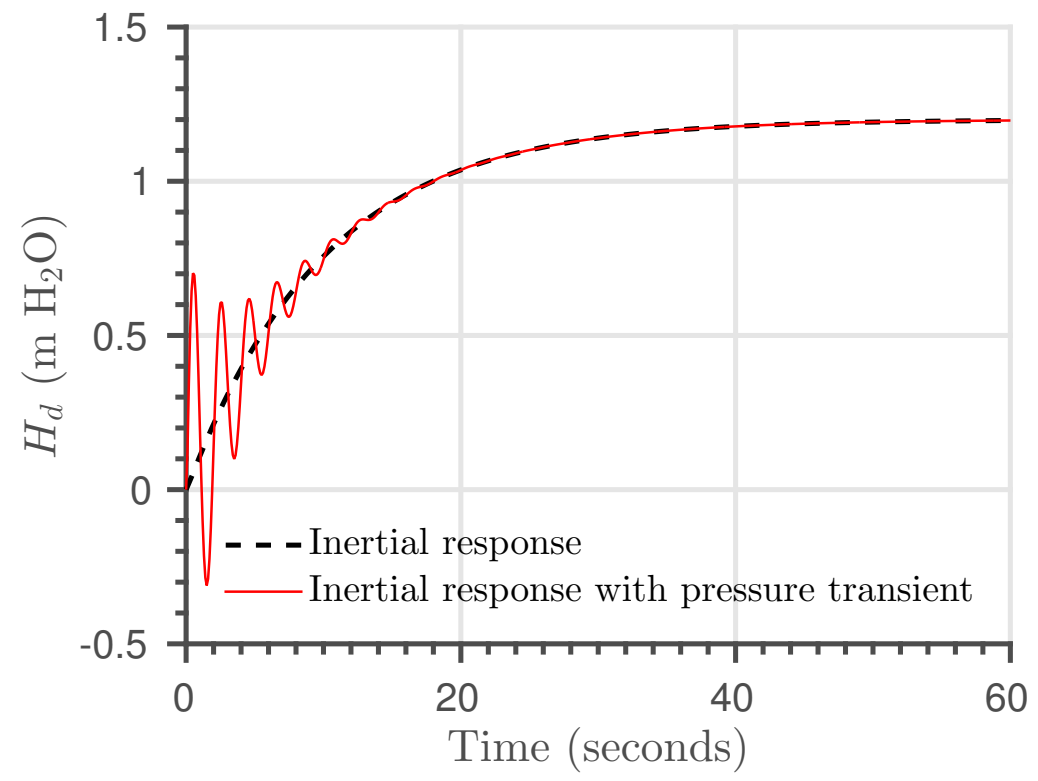

Fig. 6. Step response of lumped inertial-oscillator model of a valve/WDN to a unit step in the input. 


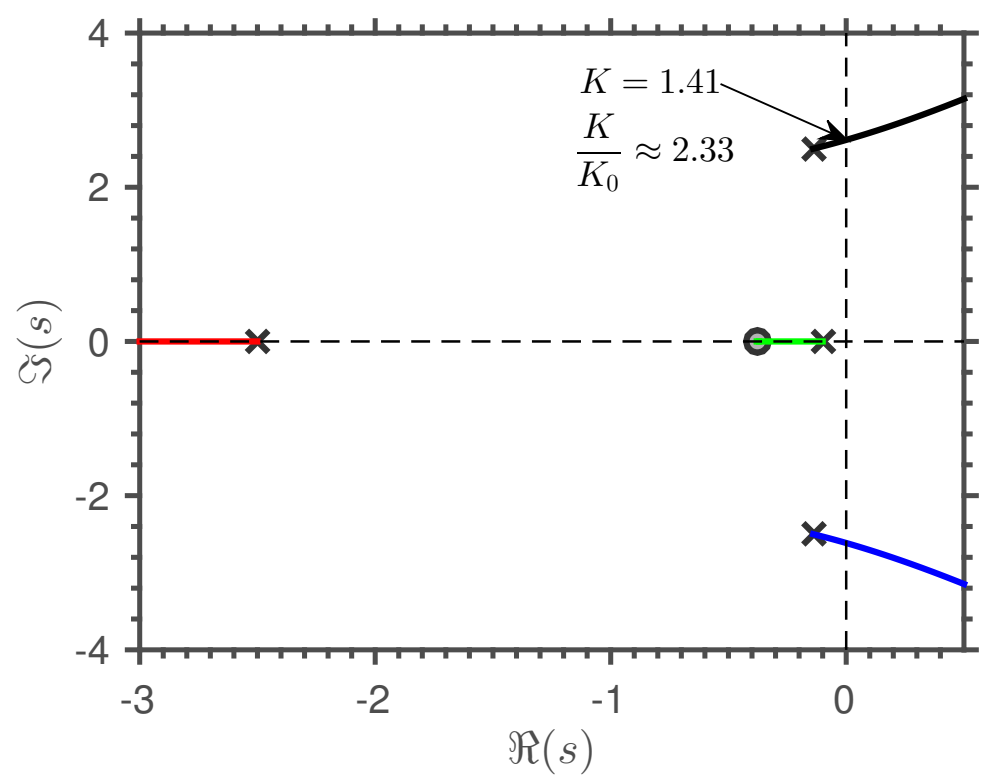

Fig. 7. Root-locus of the closed-loop system with the Maxwell model plus actuator inertia. 


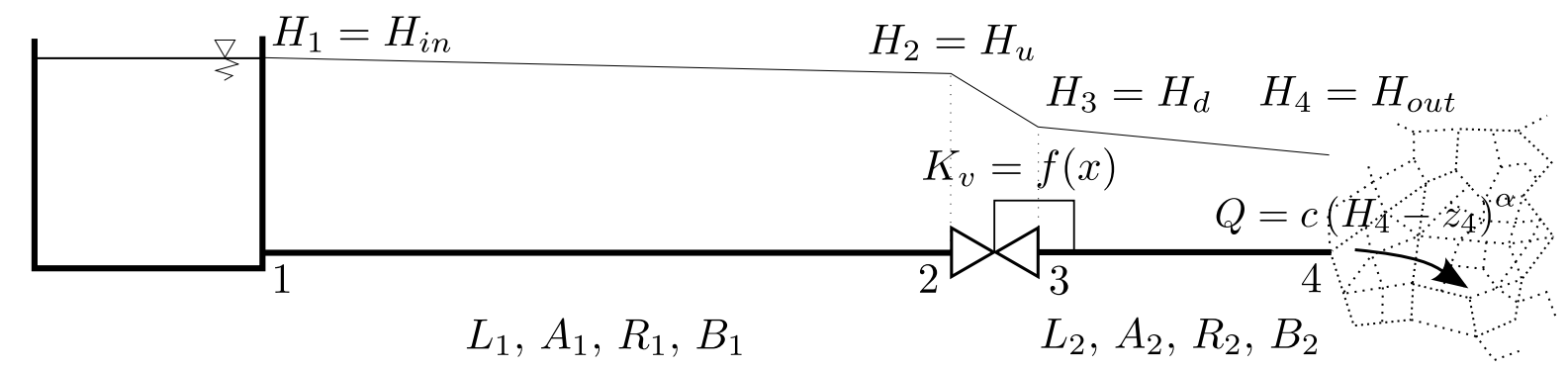

Fig. 8. Schematic of the hydraulic model used for dynamic simulation and calculation of static gain. 


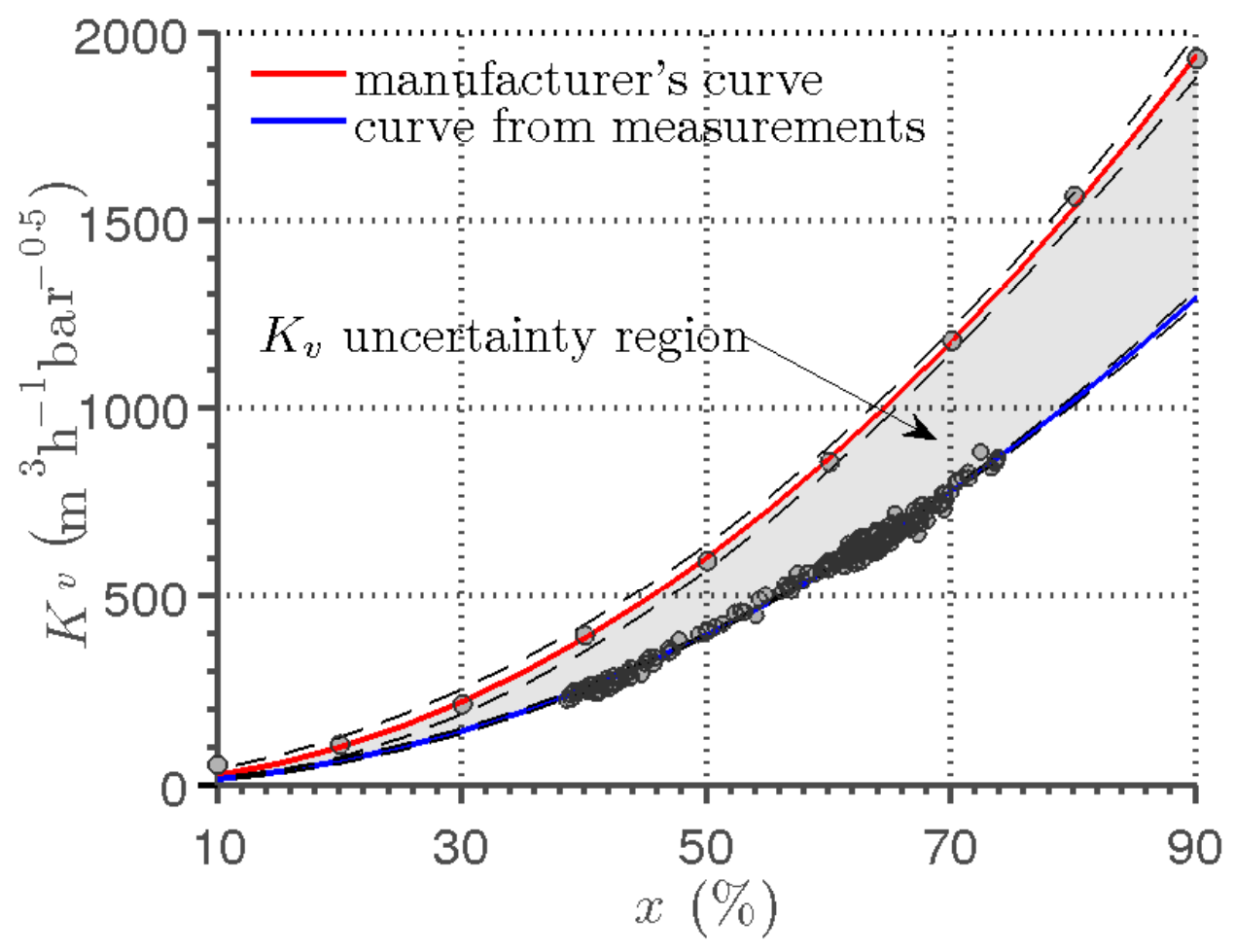

Fig. 9. Valve capacity according to the manufacturer's data and the measurements. Dashed lines denote $95 \%$ confidence intervals for the fitted curves. 


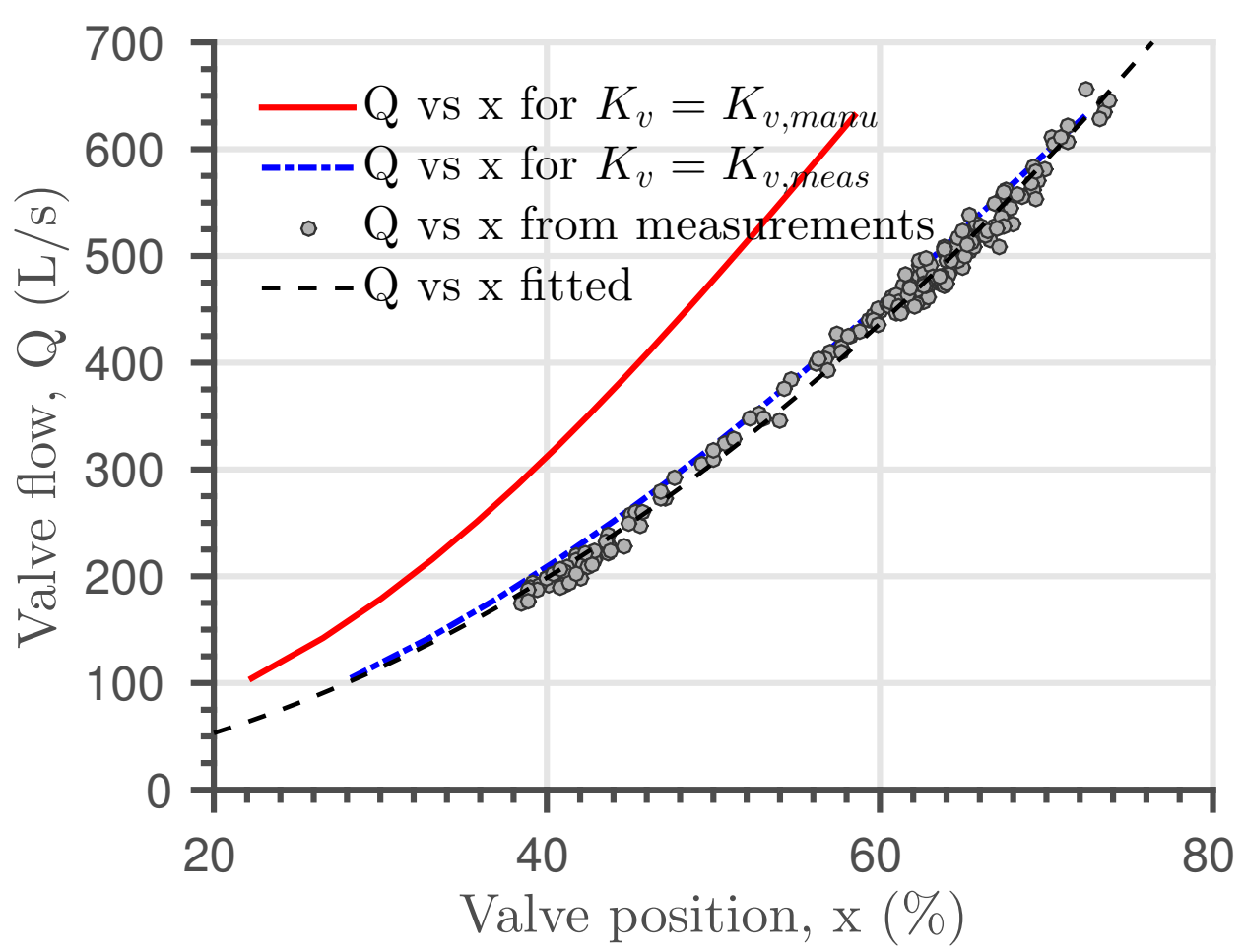

Fig. 10. Relationship between total flow $Q$ and valve position $x$ for the distribution system under study and the simplified pipe-PRV-pipe model. 


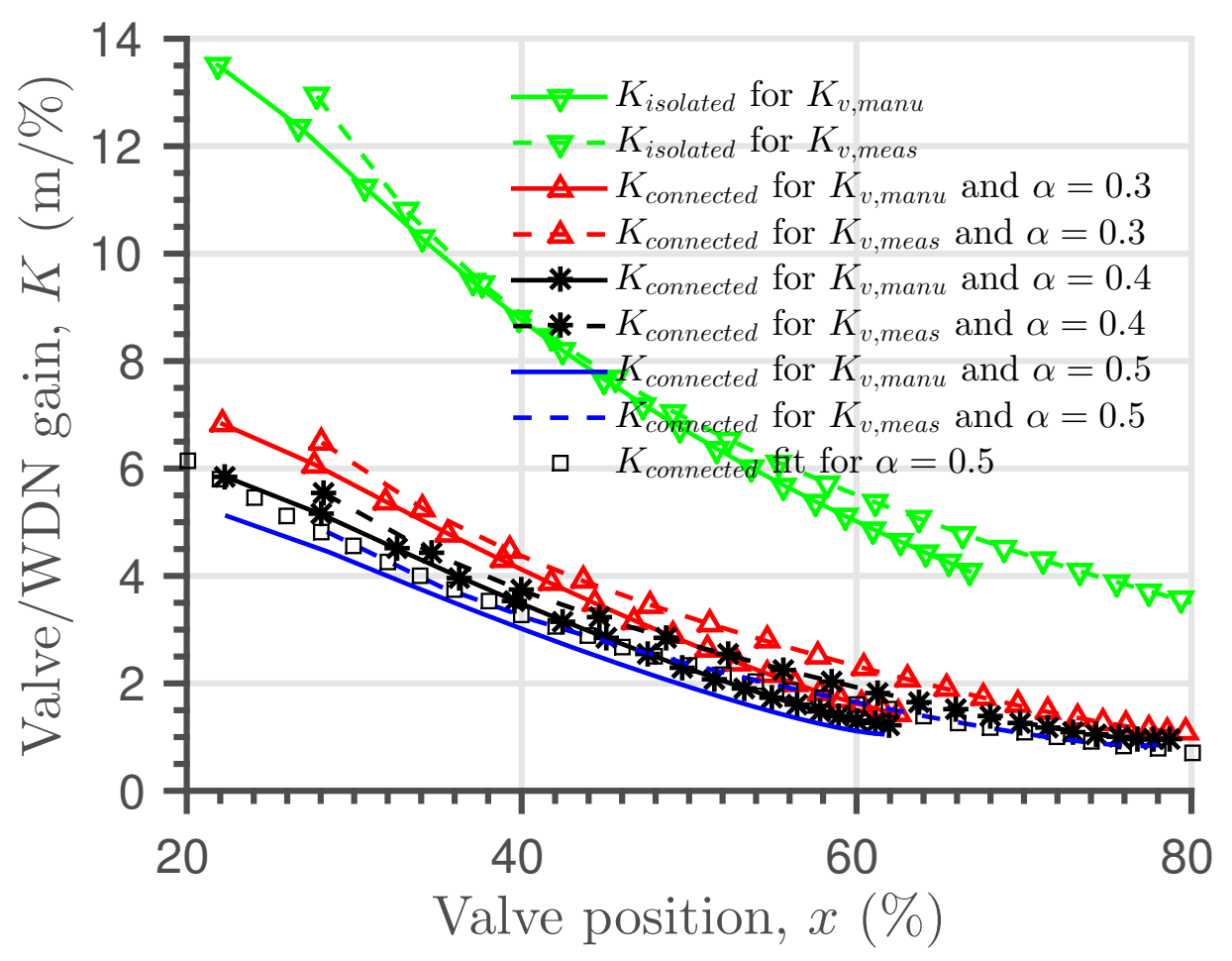

Fig. 11. Valve/WDN gain $K$ against valve position $x$ for different values of pressure dependency coefficient $\alpha$. 


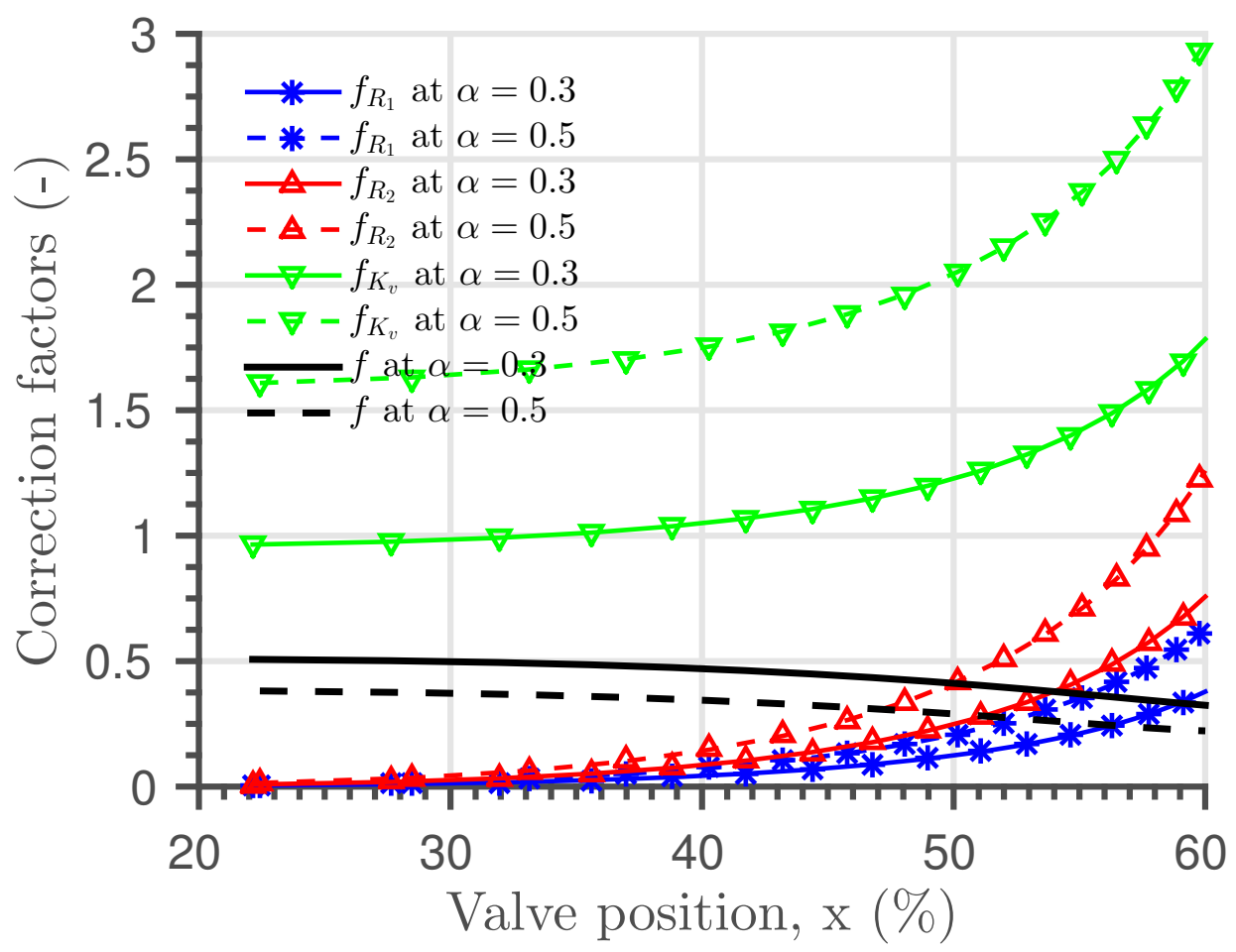

Fig. 12. Impact of $R_{1}, R_{2}$ and $K_{v}$ on the scaling function $f(\mathbf{p})$ between $K_{\text {connected }}(x)$ and $K_{\text {isolated }}(x)$. 


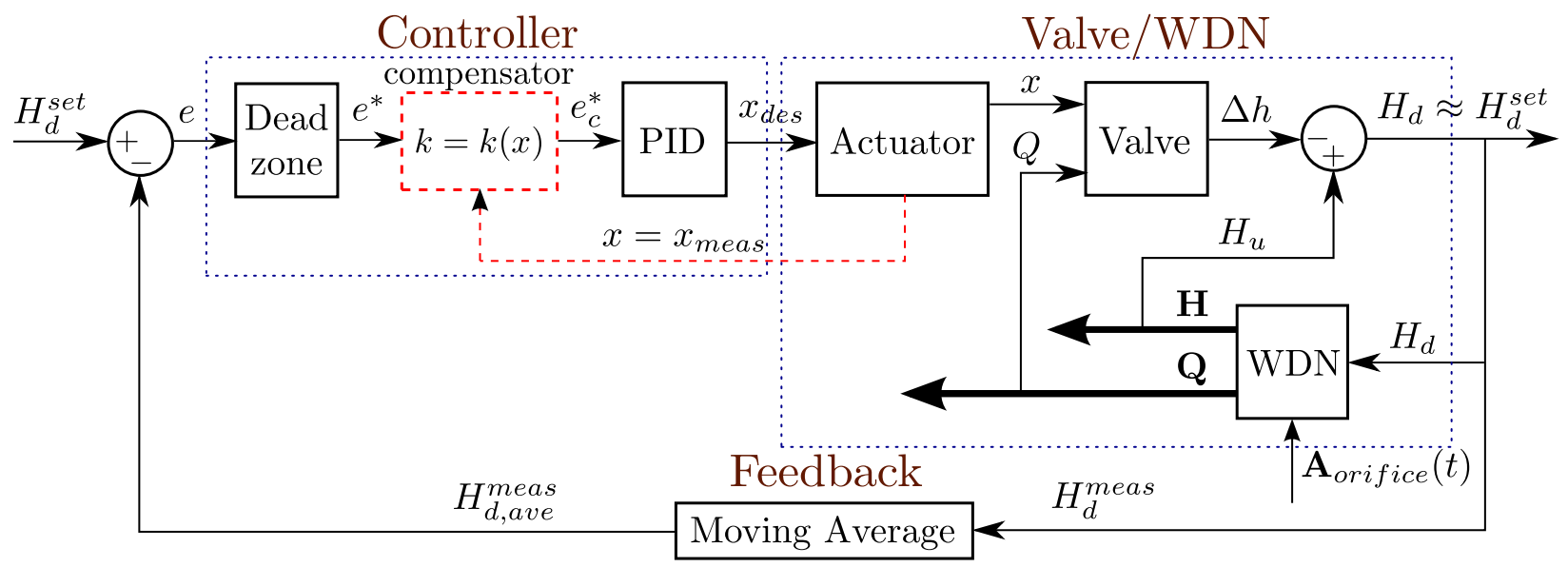

Fig. 13. Valve/WDN closed-loop model structure adopted from real-life system and implemented in the mathematical model used for the transient simulation study. 


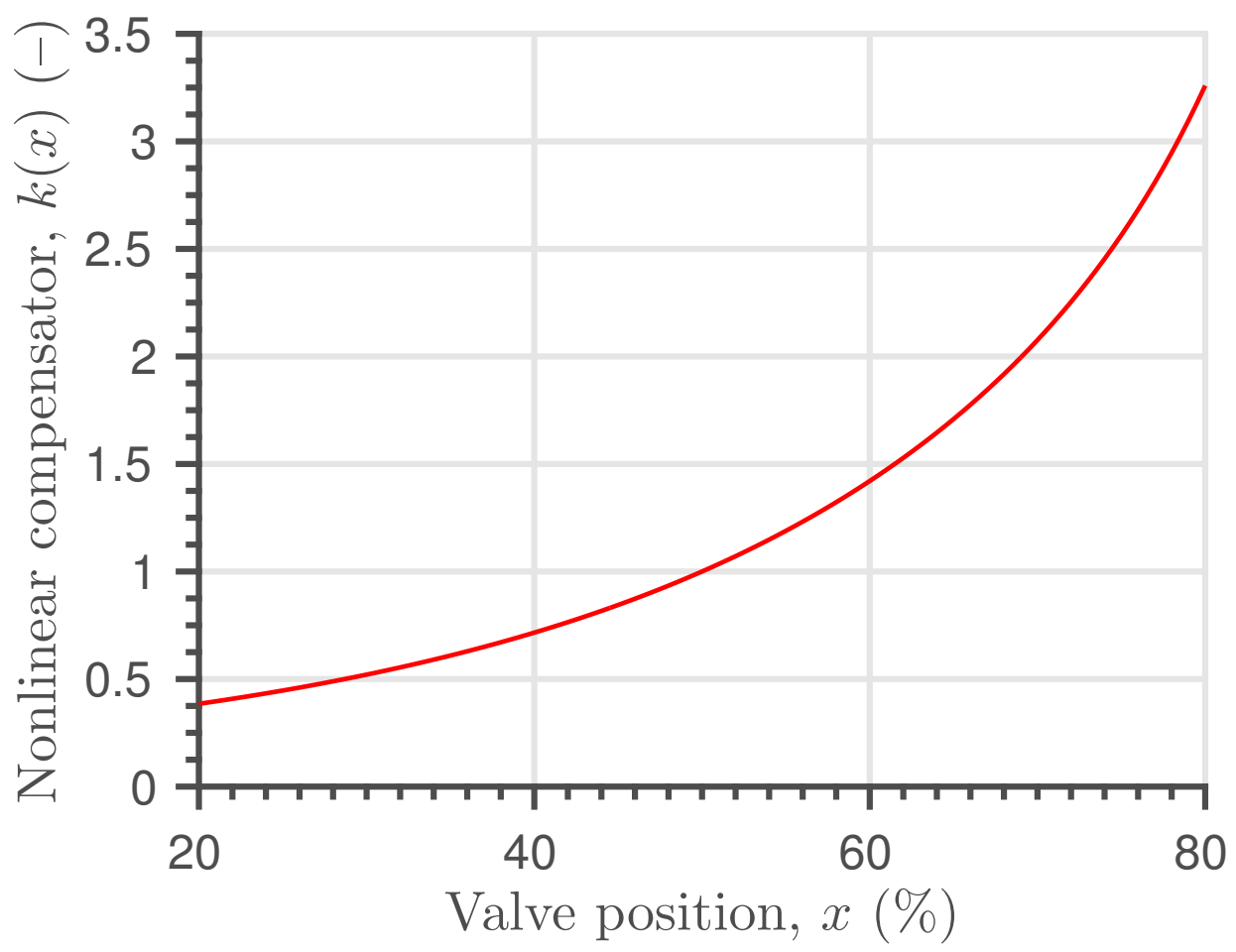

Fig. 14. Nonlinear gain compensator curve $k(x)$ used for scaling the Valve/WDN plant gain $K$ in the transient simulation study. 

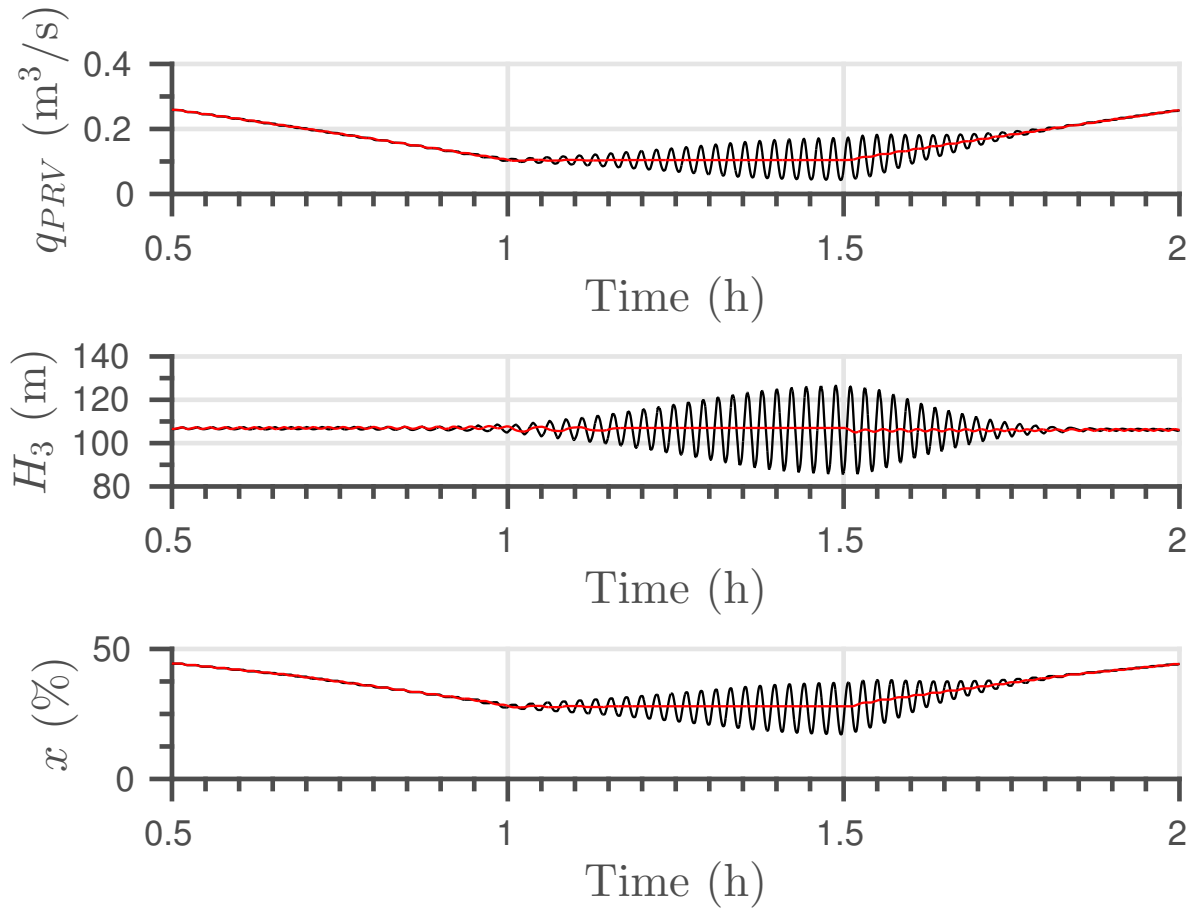

Fig. 15. Results of the transient simulations of our simplified pipe-PRV-pipe hydraulic model with (red) and without (black) gain compensation. 